\title{
On the topological cyclic homology of the algebraic closure of a local field
}

\author{
Lars Hesselholt*
}

\section{Introduction}

The cyclotomic trace map provides a comparison of the algebraic $K$-theory spectrum with a pro-spectrum $\left\{\mathrm{TR}^{n}\right\}_{n \geq 1}$ that is built from the cyclic fixed points of topological Hochschild homology. In the long paper [8] we used this comparison and an approximate evaluation of the structure of the pro-spectrum $\left\{\mathrm{TR}^{n}\right\}_{n \geq 1}$ to completely determine the $p$-adic $K$-theory of a mixed characteristic local field $K$. This verified the Lichtenbaum-Quillen conjecture for the field $K$. In this paper we completely determine the structure of the pro-spectrum $\left\{\mathrm{TR}^{n}\right\}_{n \geq 1}$ for the algebraic closure $\bar{K}$ of the local field $K$. This leads us to formulate a conjecture for the structure of the pro-spectrum $\left\{\mathrm{TR}^{n}\right\}_{n \geq 1}$ for the local field $K$.

Let $V$ be a complete discrete valuation ring with quotient field $K$ of characteristic 0 and with perfect residue field $k$ of odd characteristic $p$. In the paper [ 8 ] we constructed a map

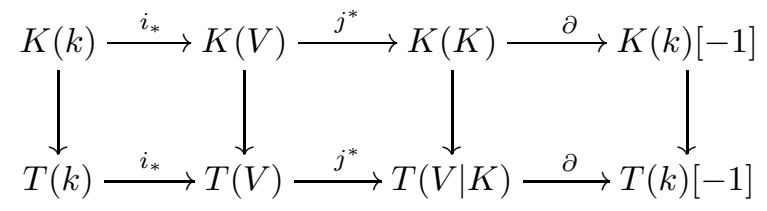

from the localization sequence in algebraic $K$-theory to an analogous cofibration sequence relating the topological Hochschild spectra $T(k)$ and $T(V)$ and a new topological Hochschild spectrum $T(V \mid K)$. The circle group $\mathbb{T}$ acts on the terms of the lower sequence, and one defines

$$
\operatorname{TR}^{n}(V \mid K ; p)=T(V \mid K)^{C_{p^{n-1}}}
$$

to be the fixed points of the subgroup $C_{p^{n-1}} \subset \mathbb{T}$ of the indicated order. As $n \geq 1$ varies, these spectra form a pro-ring-spectrum whose structure map

$$
R: \mathrm{TR}^{n}(V \mid K ; p) \rightarrow \mathrm{TR}^{n-1}(V \mid K ; p)
$$

is called the restriction map. We proved in op. cit. that the homotopy groups

$$
\operatorname{TR}_{q}^{n}(V \mid K ; p)=\pi_{q}\left(\mathrm{TR}^{n}(V \mid K ; p)\right)
$$

*) Supported in part by the National Science Foundation (USA) and COE (Japan). 
of this pro-ring-spectrum form a Witt complex over the ring $V$ endowed with the canonical log-structure given by the canonical inclusion

$$
\alpha: M=K^{*} \cap V \hookrightarrow V .
$$

We further showed that there exists an initial Witt complex $W_{n} \Omega_{(V, M)}^{q}$ over the $\log$-ring $(V, M)$ (denoted by $W_{n} \omega_{(V, M)}^{q}$ in op. cit.) and that the canonical map

$$
W_{n} \Omega_{(V, M)}^{q} \rightarrow \mathrm{TR}_{q}^{n}(V \mid K ; p)
$$

is an isomorphism, for $q \leq 2$. The main result of op. cit. was the evaluation, up to pro-isomorphism, of the homotopy groups with $\mathbb{Z} / p^{v}$-coefficients

$$
\operatorname{TR}_{q}^{n}\left(V \mid K ; p, \mathbb{Z} / p^{v}\right)=\pi_{q}\left(\operatorname{TR}^{n}(V \mid K ; p), \mathbb{Z} / p^{v}\right)
$$

in the case where the field $K$ contains the $p^{v}$ th roots of unity. Indeed, we showed in op. cit., Thm. C, that, as $n \geq 1$ varies, the canonical maps

$$
W_{n} \Omega_{(V, M)}^{q} \otimes S_{\mathbb{Z} / p^{v}}\left(\mu_{p^{v}}\right) \rightarrow \mathrm{TR}_{q}^{n}\left(V \mid K ; p, \mathbb{Z} / p^{v}\right)
$$

that take a generator $\zeta \in \mu_{p^{v}}$ to the image by the cyclotomic trace maps of the associated Bott element $\beta_{\zeta} \in K_{2}\left(K, \mathbb{Z} / p^{v}\right)$ form an isomorphism of pro-abelian groups. However, if the field $K$ does not contain the $p^{v}$ th roots of unity, the structure of the pro-abelian groups $\operatorname{TR}_{q}^{\cdot}\left(V \mid K ; p, \mathbb{Z} / p^{v}\right)$ is presently unknown.

In this paper we consider the colimit spectra

$$
\operatorname{TR}^{n}(\bar{V} \mid \bar{K} ; p)=\operatorname{colim} \operatorname{TR}^{n}\left(V_{\alpha} \mid K_{\alpha} ; p\right)
$$

where, on the right-hand side, $K_{\alpha}$ ranges over all finite field extension of $K$ that are contained in an algebraic closure $\bar{K}$ of $K$, and where $V_{\alpha}$ and $\bar{V}$ are the integral closures of $V$ in $K_{\alpha}$ and $\bar{K}$, respectively. The canonical map

$$
W_{n} \Omega_{(\bar{V}, \bar{M})}^{q} \rightarrow \operatorname{TR}_{q}^{n}(\bar{V} \mid \bar{K} ; p)
$$

is an isomorphism, for $q \leq 2$, but the statement for the higher homotopy groups with $\mathbb{Z} / p^{v}$-coefficients is not valid for $\bar{K}$ as isomorphisms of pro-abelian groups are generally not preserved under infinite colimits. The purpose of this paper is to completely determine the $p$-adic homotopy groups

$$
\operatorname{TR}_{q}^{n}\left(\bar{V} \mid \bar{K} ; p, \mathbb{Z}_{p}\right)=\pi_{q}\left(\operatorname{TR}^{n}(\bar{V} \mid \bar{K} ; p), \mathbb{Z}_{p}\right) .
$$

We recall that, for any spectrum $X$, the $p$-adic homotopy groups are related to the integral homotopy groups by a natural short-exact sequence

$$
0 \rightarrow \operatorname{Ext}\left(\mathbb{Q}_{p} / \mathbb{Z}_{p}, \pi_{q}(X)\right) \rightarrow \pi_{q}\left(X, \mathbb{Z}_{p}\right) \rightarrow \operatorname{Hom}\left(\mathbb{Q}_{p} / \mathbb{Z}_{p}, \pi_{q-1}(X)\right) \rightarrow 0 .
$$

The right-hand term is also written $T_{p}\left(\pi_{q-1}(X)\right)$ and called the $p$-primary Tate module of the group $\pi_{q-1}(X)$. It is not difficult to see that $W_{n} \Omega_{(\bar{V}, \bar{M})}^{q}$ is a divisible group, for $q$ positive, and hence we obtain a canonical isomorphism

$$
\operatorname{TR}_{2}^{n}\left(\bar{V} \mid \bar{K} ; p, \mathbb{Z}_{p}\right) \stackrel{\sim}{\rightarrow} T_{p}\left(\operatorname{TR}_{1}^{n}(\bar{V} \mid \bar{K} ; p)\right) \stackrel{\sim}{\leftarrow} T_{p}\left(W_{n} \Omega_{(\bar{V}, \bar{M})}^{1}\right) .
$$

This, in turn, induces a map of graded $\operatorname{TR}_{0}^{n}\left(\bar{V} \mid \bar{K} ; p, \mathbb{Z}_{p}\right)$-algebras

$$
S_{\mathrm{TR}_{0}^{n}\left(\bar{V} \mid \bar{K} ; p, \mathbb{Z}_{p}\right)}\left(T_{p} \operatorname{TR}_{1}(\bar{V} \mid \bar{K} ; p)\right) \rightarrow \operatorname{TR}_{*}^{n}\left(\bar{V} \mid \bar{K} ; p, \mathbb{Z}_{p}\right)
$$

by using the multiplicative structure on the right-hand side. 
Theorem A. The group $\operatorname{TR}_{q}^{n}(\bar{V} \mid \bar{K} ; p)$ is divisible, if $q>0$, and uniquely divisible, if $q>0$ and even. The p-primary Tate module $T_{p} \operatorname{TR}_{1}^{n}(\bar{V} \mid \bar{K} ; p)$ is a free module of rank one over $\operatorname{TR}_{0}^{n}\left(\bar{V} \mid \bar{K} ; p, \mathbb{Z}_{p}\right)$, and the canonical map

$$
S_{\mathrm{TR}_{0}^{n}\left(\bar{V} \mid \bar{K} ; p, \mathbb{Z}_{p}\right)}\left(T_{p} \operatorname{TR}_{1}^{n}(\bar{V} \mid \bar{K} ; p)\right) \rightarrow \operatorname{TR}_{*}^{n}\left(\bar{V} \mid \bar{K} ; p, \mathbb{Z}_{p}\right)
$$

is an isomorphism.

We note the formal analogy with the following result by Suslin [11, 12 on the algebraic $K$-theory of the field $\bar{K}$. The group $K_{q}(\bar{K})$ is divisible, if $q>0$, and uniquely divisible, if $q>0$ and even; the Tate module $T_{p} K_{1}\left(\bar{K}, \mathbb{Z}_{p}\right)$ is a free module of rank one over $K_{0}\left(\bar{K}, \mathbb{Z}_{p}\right)$, and the canonical map

$$
S_{K_{0}\left(\bar{K}, \mathbb{Z}_{p}\right)}\left(T_{p} K_{1}(\bar{K})\right) \rightarrow K_{*}\left(\bar{K}, \mathbb{Z}_{p}\right)
$$

is an isomorphism

The ring $\operatorname{TR}_{0}^{n}\left(\bar{V} \mid \bar{K} ; p, \mathbb{Z}_{p}\right)$ is canonically isomorphic to the ring $W_{n}(\bar{V})^{\wedge}$ given by the $p$-completion of the ring of ( $p$-typical) Witt vectors in $\bar{V}$. We determine the structure of this ring following the work of Fontaine 4 . To state the result, we first let $R_{\bar{V}}$ be the ring given by the limit of the diagram

$$
\bar{V} / p \bar{V} \stackrel{\varphi}{\leftarrow} \bar{V} / p \bar{V} \stackrel{\varphi}{\leftarrow} \bar{V} / p \bar{V} \stackrel{\varphi}{\leftarrow} \ldots
$$

with the Frobenius as structure map. The ring $R_{\bar{V}}$ is a perfect $\mathbb{F}_{p}$-algebra and an integrally closed domain whose quotient field is algebraically closed. We show that there is a surjective ring homomorphism

$$
\theta_{n} W\left(R_{\bar{V}}\right) \rightarrow W_{n}(\bar{V})^{\wedge}
$$

whose kernel is a principal ideal with a generator given as follows. We choose a sequence $\varepsilon=\left\{\varepsilon^{(v)}\right\}_{v \geq 1}$ of compatible primitive $p^{v-1}$ th roots of unity in $\bar{V} \subset \bar{K}$. The sequence $\varepsilon$ determines, by reduction modulo $p$, an element of $R_{\bar{V}}$ that we also denote by $\varepsilon$. We let $\varepsilon_{n} \in R_{\bar{V}}$ be the unique $p^{n}$ th root of $\varepsilon$, and let $\left[\varepsilon_{n}\right] \in W\left(R_{\bar{V}}\right)$ be the Teichmüller representative. Then $([\varepsilon]-1) /\left(\left[\varepsilon_{n}\right]-1\right)$ generates the kernel of $\theta_{n}$. Moreover, the maps $\theta_{n}$ satisfy that $R \circ \theta_{n}=\theta_{n-1}$ and $F \circ \theta_{n}=\theta_{n-1} \circ F$.

We give a similar complete description of the structure of the $p$-primary Tate module $T_{p} \operatorname{TR}_{1}^{n}(\bar{V} \mid \bar{K} ; p)$. The $K$-theory Bott element $\beta_{\varepsilon}=\beta_{\varepsilon}^{K}$ which is defined to be the image of $\varepsilon=\left\{\varepsilon^{(v)}\right\}_{v \geq 1}$ by the canonical isomorphism

$$
T_{p}\left(\bar{K}^{*}\right) \stackrel{\sim}{\rightarrow} T_{p} K_{1}(\bar{K})
$$

is a $K_{0}\left(\bar{K}, \mathbb{Z}_{p}\right)$-module generator. But the TR-theory Bott element $\beta_{\varepsilon, n}=\beta_{\varepsilon, n}^{\mathrm{TR}}$ which is defined to be the image of $\beta_{\varepsilon}$ by the cyclotomic trace map

$$
T_{p} K_{1}(\bar{K}) \rightarrow T_{p} \operatorname{TR}_{1}^{n}(\bar{V} \mid \bar{K} ; p)
$$

is not a generator of the $\operatorname{TR}_{0}^{n}\left(\bar{V} \mid \bar{K} ; p, \mathbb{Z}_{p}\right)$-module on the right-hand side. Instead we have the following result.

THEOREM B. Let $\varepsilon=\left\{\varepsilon^{(v)}\right\}_{v \geq 1}$ be a compatible sequence of $p^{v-1}$ th roots of unity in $\bar{V} \subset \bar{K}$. Then the following statements hold.

(i) The $W_{n}(\bar{V})^{\wedge}$-module $T_{p} \operatorname{TR}_{1}^{n}(\bar{V} \mid \bar{K} ; p)$ has a generator $\alpha_{\varepsilon, n}$ such that

$$
\beta_{\varepsilon, n}=\theta_{n}\left(\left[\varepsilon_{n}\right]-1\right) \cdot \alpha_{\varepsilon, n} .
$$

(ii) The restriction and Frobenius maps

$$
R, F: T_{p} \operatorname{TR}_{1}^{n}(\bar{V} \mid \bar{K} ; p) \rightarrow T_{p} \operatorname{TR}_{1}^{n-1}(\bar{V} \mid \bar{K} ; p)
$$


take $\alpha_{\varepsilon, n}$ to $\theta_{n}\left(\left(\left[\varepsilon_{n-1}\right]-1\right) /\left(\left[\varepsilon_{n}\right]-1\right)\right) \cdot \alpha_{\varepsilon, n-1}$ and $\alpha_{\varepsilon, n-1}$, respectively.

(iii) The action of the Galois group $G_{K}=\operatorname{Gal}(\bar{K} / K)$ is given by

$$
\alpha_{\varepsilon, n}^{\sigma}=\chi(\sigma) \cdot \theta_{n}\left(\left(\left[\varepsilon_{n}\right]-1\right) /\left(\left[\varepsilon_{n}^{\sigma}\right]-1\right)\right) \cdot \alpha_{\varepsilon, n},
$$

where $\chi G_{K} \rightarrow \operatorname{Aut}\left(\mu_{p \infty}\right)=\mathbb{Z}_{p}^{*}$ is the cyclotomic character.

We remark that parts (ii) and (iii) of Thm. B are easy consequences of part (i) and the fact that $R\left(\beta_{\varepsilon, n}\right)=F\left(\beta_{\varepsilon, n}\right)=\beta_{\varepsilon, n-1}$. Since in positive degrees the integral homotopy groups $\operatorname{TR}_{q}^{n}(\bar{V} \mid \bar{K} ; p)$ are divisible we obtain a canonical isomorphism

$$
\operatorname{TR}_{q}^{n}\left(\bar{V} \mid \bar{K} ; p, \mathbb{Q}_{p} / \mathbb{Z}_{p}\right) \stackrel{\sim}{\rightarrow} \operatorname{TR}_{q}^{n}\left(\bar{V} \mid \bar{K} ; p, \mathbb{Z}_{p}\right) \otimes \mathbb{Q}_{p} / \mathbb{Z}_{p} .
$$

Hence Thms. $\mathrm{A}$ and $\mathrm{B}$ also determine the structure of the $G_{K}$-modules on the left-hand side. The following conjecture was first formulated in $\underline{\mathbf{6}}$.

CONJECTURE. For all positive integers $q$, the canonical map

$$
\mathrm{TR}_{q}^{\cdot}\left(V \mid K ; p, \mathbb{Q}_{p} / \mathbb{Z}_{p}\right) \rightarrow \mathrm{TR}_{q}^{\cdot}\left(\bar{V} \mid \bar{K} ; p, \mathbb{Q}_{p} / \mathbb{Z}_{p}\right)^{G_{K}}
$$

is an isomorphism of pro-abelian groups and the higher continuous cohomology groups of the pro- $G_{K}$-module $\operatorname{TR}_{q}^{\cdot}\left(\bar{V} \mid \bar{K} ; p, \mathbb{Q}_{p} / \mathbb{Z}_{p}\right)$ vanish.

We use Thms. $\mathrm{A}$ and $\mathrm{B}$ and a theorem of Tate $\mathbf{1 3}$ to show that the rational cohomology groups $H_{\text {cont }}^{i}\left(G_{K}, \mathrm{TR}_{q}^{\cdot}\left(\bar{V} \mid \bar{K} ; p, \mathbb{Q}_{p}\right)\right)$ vanish for $i \geq 0$ and $q>0$. We hope that similar methods will make it possible to understand the structure of the cohomology groups $H_{\text {cont }}^{i}\left(G_{K}, \mathrm{TR}_{q}^{\cdot}\left(\bar{V} \mid \bar{K} ; p, \mathbb{Q}_{p} / \mathbb{Z}_{p}\right)\right)$ in question.

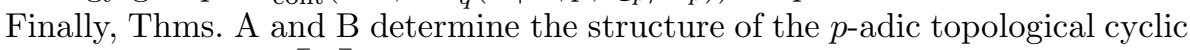
homology groups $\operatorname{TC}_{q}\left(\bar{V} \mid \bar{K} ; p, \mathbb{Z}_{p}\right)$. Indeed, we obtain the following new result.

TheOREM C. The cyclotomic trace induces an isomorphism

$$
K_{q}\left(\bar{K}, \mathbb{Z}_{p}\right) \stackrel{\sim}{\rightarrow} \mathrm{TC}_{q}\left(\bar{V} \mid \bar{K} ; p, \mathbb{Z}_{p}\right),
$$

for all integers $q$.

We remark that the common group in the statement of Thm. C is canonically isomorphic to the $G_{K}$-module $\mathbb{Z}_{p}(q / 2)$, if $q$ is a non-negative even integer, and is zero, otherwise. We also note that the vanishing of $\operatorname{TC}_{q}\left(\bar{V} \mid \bar{K} ; p, \mathbb{Z}_{p}\right)$, for $q$ odd (including $q=-1$ ), is an immediate consequence of the fact that the quotient field of the $\mathbb{F}_{p}$-algebra $R_{\bar{V}}$ is algebraically closed.

The paper is organized as follows. Sect. 1 determines the structure of the ring $W_{n}(\bar{V})^{\wedge}$; Sect. 2 contains the proofs of Thms. $\mathrm{A}$ and $\mathrm{B}$ with the exception of the existence of the generator $\alpha_{\varepsilon, n}$ which is proved in Sect. 3 and the final Sect. 4 concerns Galois cohomology.

The results of this paper were reported in expository form in [6].

It is a great pleasure to thank the University of Tokyo and Takeshi Saito in particular for their hospitality during the writing of parts of this paper. The author also expresses his sincere gratitude to an anonymous referee who read an earlier version of this paper with extreme care and made numerous helpful suggestions.

\section{Witt vectors}

1.1. In this section we determine the structure of the ring

$$
W_{n}(\bar{V})^{\wedge} \stackrel{\sim}{\rightarrow} \operatorname{TR}_{0}^{n}\left(\bar{V} \mid \bar{K} ; p, \mathbb{Z}_{p}\right)
$$


that is given by the $p$-completion of the ring of ( $p$-typical) Witt vectors of length $n$ in $\bar{V}$. This follows the work of Fontaine 4]. We refer the reader to [9, Sect. 1.1] for generalities on Witt vectors.

We say that a ring $A$ is $p$-complete if the canonical map from $A$ to the $p$ completion $A^{\wedge}=\lim _{v} A / p^{v} A$ is an isomorphism, or equivalently, if the $p$-adic topology on $A$ is complete and separated.

Lemma 1.1.1. Let $A$ be a $p$-complete and p-torsion free ring. Then $W(A)$ is again a $p$-complete and $p$-torsion free ring.

Proof. Since $A$ is $p$-torsion free then so is $W_{n}(A)$, and hence, the sequences

$$
0 \rightarrow A / p^{v} A \stackrel{V^{n-1}}{\longrightarrow} W_{n}(A) / p^{v} W_{n}(A) \stackrel{R}{\rightarrow} W_{n-1}(A) / p^{v} W_{n-1}(A) \rightarrow 0
$$

are exact. It follows, by induction, that $W_{n}(A)$ is a $p$-complete ring. Taking limits over $n$, the exact sequences

$$
0 \rightarrow W_{n}(A) \stackrel{p^{v}}{\longrightarrow} W_{n}(A) \rightarrow W_{n}(A) / p^{v} W_{n}(A) \rightarrow 0
$$

give rise to an isomorphism

$$
W(A) / p^{v} W(A) \stackrel{\sim}{\rightarrow} \lim _{n}\left(W_{n}(A) / p^{v} W_{n}(A)\right) .
$$

Taking the limit over $v$ and using that limits commute, we find that the lower horizontal map in following diagram is an isomorphism.

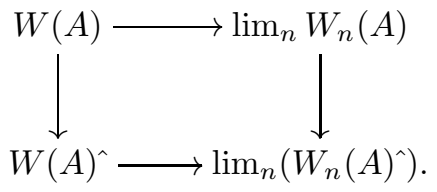

The right-hand vertical map is an isomorphism, since $W_{n}(A)$ is a $p$-complete ring, and the top horizontal map is an isomorphism for trivial reasons. Hence, the lefthand vertical map is an isomorphism as stated.

Lemma 1.1.2. Let $A$ be a ring without p-torsion. Then the canonical maps $W(A)^{\wedge} \rightarrow W\left(A^{\wedge}\right)^{\wedge}$ and $W_{n}(A)^{\wedge} \rightarrow W_{n}\left(A^{\wedge}\right)^{\wedge}$ are isomorphisms.

Proof. Since $A$ is $p$-torsion free, the canonical map $A / p^{v} A \rightarrow A^{\wedge} / p^{v} A^{\wedge}$ is an isomorphism. Hence, inductively, so are the induced maps

$$
W_{n}(A) / p^{v} W_{n}(A) \stackrel{\sim}{\rightarrow} W_{n}\left(A^{\wedge}\right) / p^{v} W_{n}\left(A^{\wedge}\right),
$$

for all $n \geq 1$. Using that $W_{n}(A)$ and $W_{n}\left(A^{\wedge}\right)$ are $p$-torsion free, we conclude that

$$
W(A) / p^{v} W(A) \stackrel{\sim}{\rightarrow} W\left(A^{\wedge}\right) / p^{v} W\left(A^{\wedge}\right) .
$$

Hence, the induced map of limits over $v$ is an isomorphism, and this is the statement of the lemma.

LEMMA 1.1.3. Let $A$ be a -complete and p-torsion free ring. Then the canonical projection

$$
\lim _{F} W(A) \rightarrow \lim _{F} W(A / p A)
$$

is an isomorphism, and the inverse is given as follows. Let $x=\left\{x^{(m)}\right\}_{m \geq 1}$ be an element of $\lim _{F} W(A / p A)$, and let $\tilde{x}^{(m)} \in W(A)$ be liftings of the individual $x^{(m)}$. Then the sequence $\left\{F^{k}\left(\tilde{x}^{(m+k)}\right)\right\}_{k \geq 0}$ converges in the $p$-adic topology to an element 
$\hat{x}^{(m)} \in W(A)$, and the sequence $\hat{x}=\left\{\hat{x}^{(m)}\right\}_{m \geq 1}$ is the element of $\lim _{F} W(A)$ whose image by the canonical projection is $x$.

Proof. We first show that the canonical projection is an isomorphism. To this end, we consider the six-term exact sequence associated with the following short-exact sequence of limit systems.

$$
0 \rightarrow W(p A) \rightarrow W(A) \rightarrow W(A / p A) \rightarrow 0 .
$$

It follows that it suffices to show that $\lim _{F} W(p A)$ and $R^{1} \lim _{F} W(p A)$ vanish. These groups are given by the kernel and cokernel, respectively, of the map

$$
\text { id }-\sigma: \prod_{m \geq 1} W(p A) \rightarrow \prod_{m \geq 1} W(p A),
$$

where $\sigma$ maps the $m$ th factor to the $(m-1)$ st factor by the Frobenius. Since $A$ is $p$-torsion free, the ghost map defines an isomorphism

$$
w: W(p A) \stackrel{\sim}{\longrightarrow} \prod_{s \geq 0} p^{s+1} A .
$$

So the map in question is isomorphic to the map

$$
\text { id }-\sigma: \prod p^{s+1} A \rightarrow \prod p^{s+1} A
$$

where the products ranges over $s \geq 0$ and $m \geq 1$ and where $\sigma$ maps the $(s, m)$ th factor to the $(s-1, m-1)$ st factor by the canonical inclusion $p^{s+1} A \rightarrow p^{s} A$. Hence, id $-\sigma$ is an isomorphism with inverse

$$
(\mathrm{id}-\sigma)^{-1}=\sum_{n \geq 0} \sigma^{n} .
$$

The series on the right converges since $A$ is $p$-complete.

It remains to show that the inverse of the canonical projection is given as stated. We must show that the sequence $\left\{F^{k}\left(\tilde{x}^{(m+k)}\right)\right\}_{k \geq 0}$ converges with a unique limit, and since the $p$-adic topology on $W(A)$ is complete and separated, it suffices to show that the sequence is Cauchy. To this end, we first note that a sequence $\left\{x_{s}\right\}_{s \geq 0}$ is in the image of the ideal $W(p A)$ by the ghost map

$$
w: W(A) \rightarrow A^{\mathbb{N}_{0}}
$$

if and only if $x_{s} \in p^{s+1} A$, for all $s \geq 0$. Now

$$
\begin{aligned}
w_{s}\left(F^{k}\left(\tilde{x}^{(m+k)}\right)\right) & =w_{s+k}\left(\tilde{x}^{(m+k)}\right) \\
& =\left(\tilde{x}_{0}^{(m+k)}\right)^{p^{s+k}}+p\left(\tilde{x}_{1}^{(m+k)}\right)^{p^{s+k-1}}+\cdots+p^{s+k} \tilde{x}_{s+k}^{(m+k)}
\end{aligned}
$$

which shows that

$$
w_{s}\left(F^{k+1}\left(\tilde{x}^{(m+k+1)}\right)\right)-w_{s}\left(F^{k}\left(\tilde{x}^{(m+k)}\right)\right) \in p^{s+k+1} A .
$$

Hence the sequence $\left\{F^{k}\left(\tilde{x}^{(m+k)}\right)\right\}_{k \geq 0}$ is Cauchy as desired.

Lemma 1.1.4. Let $A$ be a p-complete and p-torsion free ring. Then the canonical projection induces an isomorphism

$$
\lim _{F} W(A) \stackrel{\sim}{\rightarrow} \lim _{F} W_{n}(A) .
$$


Proof. We consider the exact sequence of limit systems with $n$th terms

$$
0 \rightarrow W(A) \stackrel{V^{n}}{\longrightarrow} W(A) \stackrel{\mathrm{pr}}{\longrightarrow} W_{n}(A) \rightarrow 0 .
$$

The structure maps in the middle and right-hand limit systems are given by the Frobenius, and the structure map in the left-hand limit system is given by multiplication by $p$. The induced six-term exact sequence takes the form

$$
\begin{aligned}
0 & \rightarrow \lim _{p} W(A) \rightarrow \lim _{F} W(A) \rightarrow \lim _{F} W_{n}(A) \\
& \rightarrow R^{1} \lim _{p} W(A) \rightarrow R^{1} \lim _{F} W(A) \rightarrow R^{1} \lim _{F} W_{n}(A) \rightarrow 0 .
\end{aligned}
$$

The first and fourth terms vanish since the $p$-adic topology on $W(A)$ is separated and complete, respectively. The lemma follows.

1.2. Let $V$ be a complete discrete valuation ring with quotient field $K$ and perfect residue field $k$ of mixed characteristic $(0, p)$. Let $\bar{K}$ be an algebraic closure of $K$ and let $\bar{V}$ be the integral closure of $V$ in $\bar{K}$. Let

$$
\bar{V}^{\wedge}=\lim _{n} \bar{V} / p^{n} \bar{V}
$$

be the $p$-completion of $\bar{V}$, and let $\bar{K}^{\wedge}$ be the quotient field of $\bar{V}^{\wedge}$. Then $\bar{K}^{\wedge}$ is again algebraically closed 1, Chap. 2, Thm. 12]. The valuation on $K$ extends uniquely to a valuation on $\bar{K}$ (resp. $\bar{K}^{\wedge}$ ) with value group the additive group of rational numbers and $\bar{V}$ (resp. $\bar{V}^{\wedge}$ ) is the valuation ring. In particular, $\bar{V}$ (resp. $\bar{V}^{\wedge}$ ) is an integrally closed local ring of dimension one. However, $\bar{V}$ (resp. $\bar{V}^{\wedge}$ ) is not a noetherian ring. We normalize the valuation on $\bar{V}$ (resp. $\left.\bar{V}^{\wedge}\right)$ such that $v(p)=1$.

Let $x, y \in \bar{V}$ and suppose that $x^{p}=y^{p}$. Then $v(x-y) \geq 1 /(p-1)$. Indeed, we have $x=\zeta y$ with $\zeta^{p}=1$, and hence, $x-y=(\zeta-1) y$. But $\zeta-1$ is either zero or a uniformizer of $\mathbb{Q}_{p}\left(\mu_{p}\right)$, and thus, $v(\zeta-1) \geq 1 /(p-1)$.

We follow [9, Sect. 1.1] and write $[a]_{n} \in W_{n}(A)$ for the Teichmüller representative of $a \in A$. We note that the notation $\underline{a}_{n}$ was used instead of $[a]_{n}$ in $[\mathbf{8}$.

LEMma 1.2.1. The map $\theta_{n}^{\prime}: \bar{V} \rightarrow W_{n}(\bar{V}) / p W_{n}(\bar{V})$ given by $\theta_{n}^{\prime}(x)=[x]_{n}^{p}$ is a surjective ring homomorphism whose kernel is the ideal of elements of valuation greater than or equal to $\left(1-p^{-n}\right) /(p-1)$. Moreover, $R\left(\theta_{n}^{\prime}(x)\right)=\theta_{n-1}^{\prime}(x)$, $F\left(\theta_{n}^{\prime}(x)\right)=\theta_{n-1}^{\prime}\left(x^{p}\right)$, and $V\left(\theta_{n-1}^{\prime}(x)\right)=\theta_{n}^{\prime}\left((-p x)^{p^{-1}}\right)$.

Proof. We recall from [8, Lemma 3.1.2] that for every ring $A$, and for all $x, y \in A$, we have

$$
[x]_{n}^{p}+[y]_{n}^{p}=\left([x]_{n}+[y]_{n}\right)^{p}=\left([x+y]_{n}\right)^{p}
$$

in $W_{n}(A) / p W_{n}(A)$. Moreover, if $p$ is odd, then in addition

$$
V(1)=[-p]_{n}
$$

in $W_{n}(A) / p W_{n}(A)$ by op. cit., Lemma 3.1.1. It follows that the map $\theta_{n}^{\prime}$ is a ring homomorphism and that $\theta_{n}^{\prime}\left((-p)^{p^{-1}}\right)=V(1)$. By easy induction, we find

$$
\theta_{n}^{\prime}\left(a^{p^{-(i+1)}}(-p)^{\left(1-p^{-i}\right) /(p-1)}\right)=[a]_{n}^{p^{-i}} V^{i}(1)=V^{i}\left([a]_{n-i}\right),
$$

which shows that $\theta_{n}^{\prime}$ is surjective. Moreover, $\theta_{n}^{\prime}(x) \in V^{i} W_{n}(\bar{V}) / p V^{i} W_{n}(\bar{V})$ if and only if $v(x) \geq\left(1-p^{-i}\right) /(p-1)$.

Corollary 1.2.2. The Frobenius $F: W_{n}(\bar{V})^{\wedge} \rightarrow W_{n-1}(\bar{V})^{\wedge}$ is surjective. 
Proof. It follows from Lemma 1.2.1 that the statement holds after reduction modulo $p$. Let $W_{n, v}(\bar{V})=W_{n}(\bar{V}) / p^{v} W_{n}(\bar{V})$. Then an induction argument based on the diagram

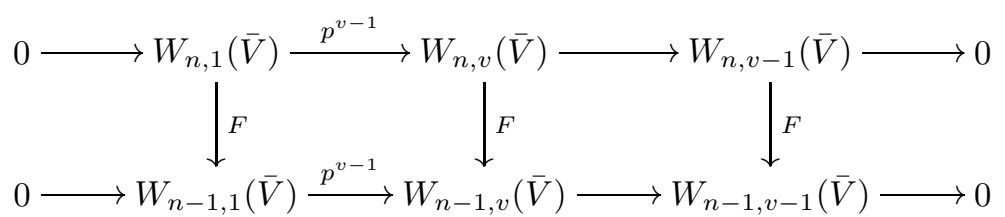

shows that the statement is true after reduction modulo $p^{v}$, for all $v \geq 1$. Since the vertical maps in the diagram above are all surjective, the sequence of kernels

$$
0 \rightarrow K_{n, 1} \rightarrow K_{n, v} \rightarrow K_{n, v-1} \rightarrow 0
$$

is exact. Hence, the sequence of limit systems

$$
0 \rightarrow K_{n, v} \rightarrow W_{n, v}(\bar{V}) \stackrel{F}{\rightarrow} W_{n-1, v}(\bar{V}) \rightarrow 0
$$

gives an exact sequence

$$
0 \rightarrow \lim _{v} K_{n, v} \rightarrow \lim _{v} W_{n, v}(\bar{V}) \stackrel{F}{\rightarrow} \lim _{v} W_{n-1, v}(\bar{V}) \rightarrow 0 .
$$

The derived limit $R^{1} \lim _{v} K_{n, v}$ vanishes since the structure maps are surjective.

Following Fontaine [4] we define $R_{\bar{V}}$ to be the limit of the diagram

$$
\cdots \rightarrow \bar{V} / p \bar{V} \stackrel{\varphi}{\rightarrow} \bar{V} / p \bar{V} \stackrel{\varphi}{\rightarrow} \bar{V} / p \bar{V}
$$

where $\varphi$ is the Frobenius. We note that the map of limits induced from the following map of towers of multiplicative monoids is an isomorphism.

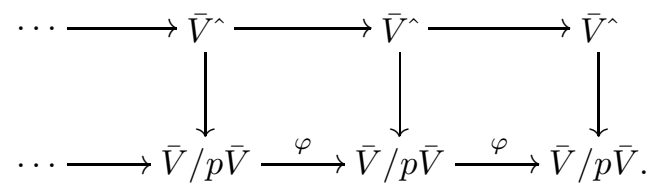

The horizontal maps are given by raising to the $p$ th power. Indeed, given an element $x=\left\{x^{(v)}\right\}_{v \geq 1} \in R_{\bar{V}}$ we choose liftings $\tilde{x}^{(v)} \in \bar{V}$ of $x^{(v)}$. Then the sequence $\left\{\left(\tilde{x}^{(m+v)}\right)^{p^{m}}\right\}_{m \geq 0}$ converges in $\bar{V}^{\wedge}$ and the limit

$$
\hat{x}^{(v)}=\lim _{m \rightarrow \infty}\left(\tilde{x}^{(m+v)}\right)^{p^{m}}
$$

is independent of the choice of liftings $\tilde{x}^{(v)}$ of $x^{(v)}$. We define a valuation $v_{R}$ of $R_{\bar{V}}$ by $v_{R}(x)=v_{\bar{V}^{\wedge}}\left(\hat{x}^{(1)}\right)$. We note that the kernel of the canonical projection

$$
\operatorname{pr}_{1}: R_{\bar{V}} \rightarrow \bar{V} / p \bar{V}
$$

is equal to the ideal of elements of valuation greater than or equal to 1.

Proposition 1.2.3. There is a natural surjective ring homomorphism

$$
\theta_{n}: W\left(R_{\bar{V}}\right) \rightarrow W_{n}(\bar{V})^{\wedge}
$$

whose kernel is a principal ideal. If $\varepsilon=\left\{\varepsilon^{(v)}\right\}_{v \geq 1}$ is a compatible sequence of primitive $p^{v-1}$ th roots of unity in $\bar{V}$ considered as an element of $R_{\bar{V}}$, and if $\varepsilon_{n}$ is the unique $p^{n}$ th root of $\varepsilon$, then $([\varepsilon]-1) /\left(\left[\varepsilon_{n}\right]-1\right)$ generates the kernel of $\theta_{n}$. Moreover, the maps $\theta_{n}$ satisfy that $R \circ \theta_{n}=\theta_{n-1}$ and $F \circ \theta_{n}=\theta_{n-1} \circ F$. 
ProOF. We consider the ring isomorphism

$$
\psi: W\left(R_{\bar{V}}\right) \rightarrow \lim _{F} W_{n}(\bar{V})^{\wedge}
$$

defined to be the composite of the following isomorphisms.

$$
W\left(R_{\bar{V}}\right) \stackrel{\sim}{\rightarrow} \lim _{F} W(\bar{V} / p \bar{V}) \stackrel{\sim}{\leftarrow} \lim _{F} W(\bar{V})^{\wedge} \stackrel{\sim}{\rightarrow} \lim _{F} W_{n}(\bar{V})^{\wedge} .
$$

The first map is an isomorphism since the underlying set of the Witt ring $W(A)$ is the product of copies of $A$ indexed by the set of non-negative integers; the second map is an isomorphism by Lemma 1.1.3 and the third map is an isomorphism by Lemma 1.1.4 The map of the statement is then induced from the composite

$$
W\left(R_{\bar{V}}\right) \stackrel{F^{n-1}}{\longrightarrow} W\left(R_{\bar{V}}\right) \stackrel{\psi}{\rightarrow} \lim _{F} W_{n}(\bar{V})^{\wedge} \stackrel{\mathrm{pr}_{n}}{\longrightarrow} W_{n}(\bar{V})^{\wedge} .
$$

We first show that $\theta_{n}\left(\xi_{n}\right)=0$. The map $F^{n-1}$ takes $\left[\varepsilon_{m}\right]$ to $\left[\varepsilon_{m-(n-1)}\right]$, and the canonical isomorphism

$$
W\left(R_{\bar{V}}\right)=W\left(\lim _{\varphi} \bar{V} / p \bar{V}\right) \stackrel{\sim}{\rightarrow} \lim _{F} W(\bar{V} / p \bar{V})
$$

takes $\left[\varepsilon_{k}\right]$ to the sequence $\left\{\left[\varepsilon_{k}^{(v)}\right]\right\}_{v \geq 1}$. To find the image of this sequence by the inverse of the canonical isomorphism

$$
\lim _{F} W(\bar{V} / p \bar{V}) \stackrel{\sim}{\leftarrow} \lim _{F} W(\bar{V})^{\wedge},
$$

we use the formula given by Lemma 1.1.3 but with a particular choice of liftings $\tilde{x}^{(v)}$ to $W(\bar{V})^{\wedge}$ of the elements $\left[\varepsilon_{k}^{(v)}\right]$ of $W(\bar{V} / p \bar{V})$. Let $\tilde{\varepsilon}_{k}=\left\{\tilde{\varepsilon}_{k}^{(v)}\right\}_{v \geq 1}$ be a sequence of elements in $\bar{V}$ such that $\varepsilon_{k}^{(v)}$ is a primitive $p^{k+v-1}$ th root of unity, for $k+v-1$ nonnegative, and such that $\left(\varepsilon_{k}^{(v+1)}\right)^{p}=\varepsilon_{k}^{(v)}$, for all $v \geq 1$. Then we choose $\tilde{x}_{k}^{(v)}=\left[\tilde{\varepsilon}_{k}^{(v)}\right]$. Since these elements are already compatible under the Frobenius map, we find that also $\hat{x}_{k}^{(v)}=\left[\tilde{\varepsilon}_{k}^{(v)}\right]$. Hence, the image of the sequence $\left\{\left[\varepsilon_{k}^{(v)}\right]\right\}_{v \geq 1}$ by the inverse of the isomorphism above, is the sequence $\left\{\left[\tilde{\varepsilon}_{k}^{(v)}\right]\right\}_{v \geq 1}$. But this sequence is mapped to 1 by the composite

$$
\lim _{F} W(\bar{V})^{\wedge} \stackrel{\sim}{\rightarrow} \lim _{F} W_{n}(\bar{V})^{\wedge} \stackrel{\mathrm{pr}}{\longrightarrow} W_{n}(\bar{V})^{\wedge}
$$

if and only if $k \leq-(n-1)$. In particular, $\theta_{n}\left(\xi_{n}\right)$ is zero as stated.

It remains to show that $\xi_{n}$ generates the kernel of $\theta_{n}$. We show inductively that for all $v \geq 1$, the induced map

$$
\theta_{n, v}: W_{v}\left(R_{\bar{V}}\right) /\left(\xi_{n, v}\right) \rightarrow W_{n}(\bar{V}) / p^{v} W_{n}(\bar{V})
$$

is an isomorphism. Here $\xi_{n, v}=\left([\varepsilon]_{v}-1\right) /\left(\left[\varepsilon_{n}\right]_{v}-1\right)$ is the image by the canonical projection of $\xi_{n}$ in $W_{v}\left(R_{\bar{V}}\right)$. In the basic case $v=1$, we have the following commutative diagram.

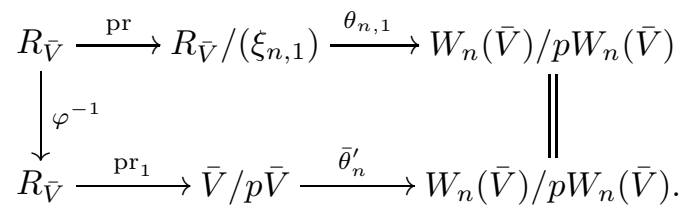

By Lemma 1.2.1 the kernel of the composition of the maps in the lower row is equal to the ideal of elements of valuation greater than or equal to $\left(1-p^{-n}\right) /(p-1)$. The 
element $\xi_{n, 1}$, which generates the kernel of the composition of the maps in the top two, has valuation

$$
v_{R}\left(\xi_{n, 1}\right)=v_{R}(\varepsilon-1)-v_{R}\left(\varepsilon_{n}-1\right)=\frac{1}{p^{-1}(p-1)}-\frac{1}{p^{n-1}(p-1)}=\frac{1-p^{-n}}{1-p^{-1}} .
$$

Since $\varphi^{-1}$ is an isomorphism and $v_{R}\left(\varphi^{-1}(x)\right)=p^{-1} v_{R}(x)$, it follows that $\theta_{n, 1}$ is an isomorphism as desired.

Finally, to prove the induction step it suffices to show that the top row in the following diagram is exact.

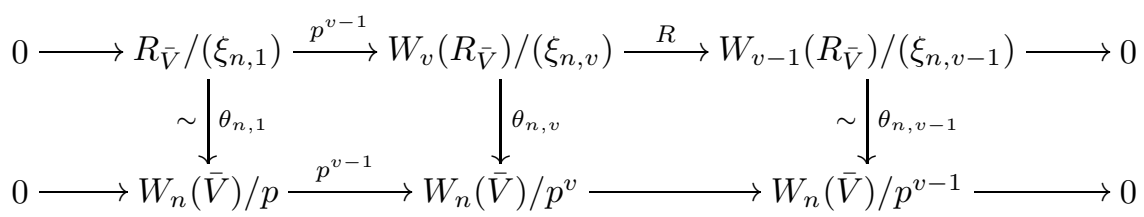

But the sequence

$$
0 \longrightarrow R_{\bar{V}} \stackrel{p^{n-1}}{\longrightarrow} W_{v}\left(R_{\bar{V}}\right) \stackrel{R}{\longrightarrow} W_{v-1}\left(R_{\bar{V}}\right) \longrightarrow 0
$$

is exact, since $R_{\bar{V}}$ is a perfect $\mathbb{F}_{p}$-algebra, and the element $\xi_{n, 1} \in R_{\bar{V}}$ is a non-zerodivisor. It follows, inductively, that $\xi_{n, v} \in W_{v}\left(R_{\bar{V}}\right)$ is a non-zero-divisor and that the sequence in question is exact.

ADDENDUM 1.2.4. There is a ring isomorphism

$$
\psi: W\left(R_{\bar{V}}\right) \stackrel{\sim}{\rightarrow} \lim _{F} W_{n}(\bar{V})^{\wedge}
$$

given by $\psi(a)=\left\{\theta_{n}\left(F^{-(n-1)}(a)\right)\right\}_{n \geq 1}$. Moreover, $R \circ \psi=\psi \circ F^{-1}$.

Proof. We showed at the beginning of the proof of Prop. 1.2.3 that the map $\psi$ is an isomorphism. The statement that $R \circ \psi=\psi \circ F^{-1}$ follows easily from the formula $R \circ \theta_{n}=\theta_{n-1}$.

1.3. We conclude this section with some miscellaneous results about the Witt rings $W\left(R_{\bar{V}}\right)$ and $W_{n}(\bar{V})^{\wedge}$. The following result is [4, 1.3.1].

Proposition 1.3.1. The ring $R_{\bar{V}}$ is a perfect $\mathbb{F}_{p}$-algebra and an integrally closed domain whose quotient field is algebraically closed and complete with respect to the valuation $v_{R}$ with valuation ring $R_{\bar{V}}$.

AdDendum 1.3.2. The ring $W\left(R_{\bar{V}}\right)$ is an integral domain.

Proof. It suffices, by Prop. 1.3.1 to show that if $A$ is an $\mathbb{F}_{p}$-algebra and an integral domain, then $W(A)$ is an integral domain. Suppose, conversely, that $W(A)$ is not an integral domain. We show that we can find a zero-divisor $x \in W(A)$ such that $x+V W(A)$ is a zero-divisor in $A=W(A) / V W(A)$. Let $x, y \in W(A)$ be non-zero and suppose $x y=0$. We write $x=V^{s}\left(x^{\prime}\right)$ and $y=V^{t}\left(y^{\prime}\right)$ with $s$ and $t$ as large as possible. Assuming that $s \geq t$,

$$
x y=V^{s}\left(x^{\prime}\right) V^{t}\left(y^{\prime}\right)=V^{s}\left(x^{\prime} F^{s} V^{t}\left(y^{\prime}\right)\right)=p^{t} V^{s}\left(x^{\prime} F^{s-t}\left(y^{\prime}\right)\right) .
$$

We claim that $W(A)$ has no $p$-torsion. Granting this, we see that $x^{\prime}+V W(A)$ is a zero-divisor in $A=W(A) / V W(A)$. It remains to prove the claim. Since $A$ is an $\mathbb{F}_{p}$-algebra, we have $p=V(1)$, and hence, for $a \in W(A), p a=V(1) a=V(F(a))$. We must show that $F: W(A) \rightarrow W(A)$ is a monomorphism. Again since $A$ is 
an $\mathbb{F}_{p}$-algebra, $F$ is induced from the Frobenius $\varphi: A \rightarrow A$. But $\varphi: A \rightarrow A$ is a monomorphism since $A$ is a domain.

COROLlary 1.3.3. Let $\varepsilon=\left\{\varepsilon^{(v)}\right\}_{v \geq 1}$ be a compatible sequence of primitive $p^{v-1}$ th roots of unity considered as an element of $R_{\bar{V}}$, and let $\varepsilon_{n}$ be the unique $p^{n}$ th root of $\varepsilon$. Then for every non-negative integer $q$, the following sequence is exact.

$$
0 \rightarrow W\left(\mathbb{F}_{p}\right) \stackrel{\left(\left[\varepsilon_{1}\right]-1\right)^{q}}{\longrightarrow} W\left(R_{\bar{V}}\right) \stackrel{1-\left(\frac{\left[\varepsilon_{1}\right]-1}{\left[\varepsilon_{2}\right]-1}\right)^{q} W\left(\varphi^{-1}\right)}{\longrightarrow} W\left(R_{\bar{V}}\right) \rightarrow 0
$$

Proof. Let $a=\left(\left[\varepsilon_{1}\right]_{n}-1\right) /\left(\left[\varepsilon_{2}\right]_{n}-1\right)$. Then the zeroth Witt coordinate is $a_{0}=a(0)=\left(\varepsilon_{1}-1\right) /\left(\varepsilon_{2}-1\right)$. We show by induction on $n \geq 1$ that the sequences

$$
0 \longrightarrow W_{n}\left(\mathbb{F}_{p}\right) \stackrel{\left[\varepsilon_{1}\right]_{n}-1}{\longrightarrow} W_{n}\left(R_{\bar{V}}\right) \stackrel{1-a W_{n}\left(\varphi^{-1}\right)}{\longrightarrow} W_{n}\left(R_{\bar{V}}\right) \longrightarrow 0
$$

are exact. The basic case $n=1$ and the induction step are similar. Indeed, it will suffice to show that for all $n \geq 1$, the top horizontal sequence in the following diagram is exact.

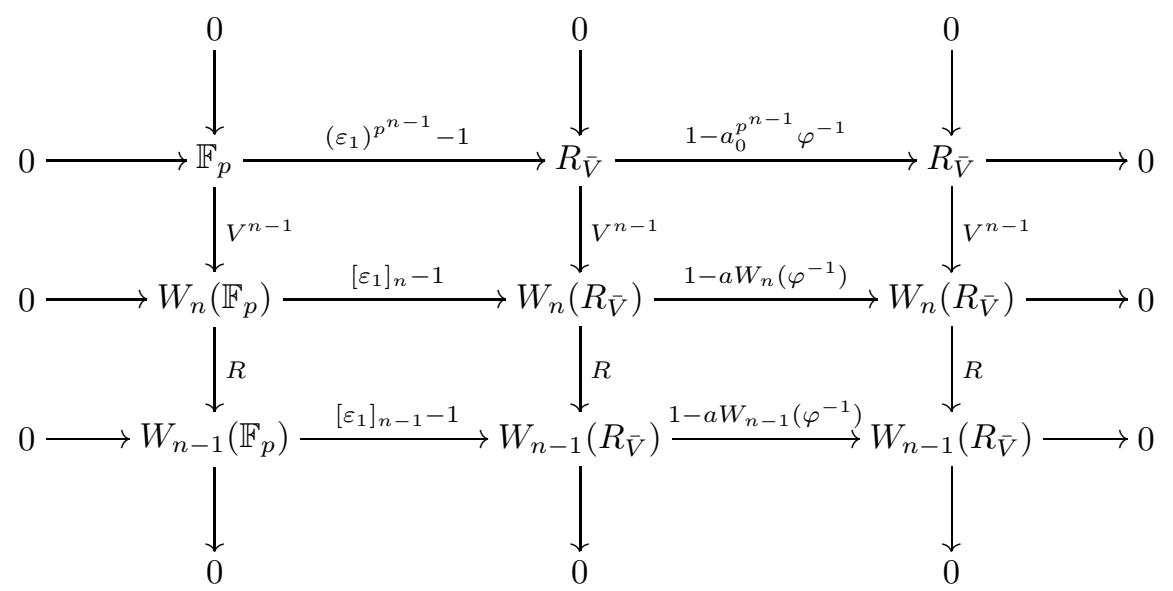

We mention that the upper right-hand square commutes since

$$
a V^{n-1}(x)=V^{n-1}\left(F^{n-1}(a) x\right)=V^{n-1}\left(a_{0}^{p^{n-1}} x\right)
$$

and similar for the upper left-hand square. To prove that the top horizontal sequence in the diagram above is exact we compose the right hand map in the sequence with the isomorphism $\varphi: R_{\bar{V}} \rightarrow R_{\bar{V}}$. The composite map takes $x$ to $x^{p}-a_{0}^{p^{n}} x$. Since $R_{\bar{V}}$ is an integrally closed domain whose quotient field is algebraically closed, the exactness follows.

LEMMA 1.3.4. The $p^{m}$ th roots of unity in the ring $W_{n}(\bar{V})^{\wedge}$ are the $p^{m}$ elements of the form $[\zeta]_{n}$ where $\zeta$ is a $p^{m}$ th root of unity in the ring $\bar{V}^{\wedge}$.

PROOF. The number of $p^{m}$ th roots of unity in the ring $\bar{V}^{\wedge}$ is equal to $p^{m}$. Hence it suffices to show that every $p^{m}$ th root of unity in $W_{n}(\bar{V})^{\wedge}$ is of the form $[\zeta]_{n}$ where $\zeta$ is a $p^{m}$ th root of unity in $\bar{V}^{\wedge}$. Now an element $a \in W_{n}(\bar{V})^{\wedge}$ is a $p^{m}$ th root of unity if an only if the image of $a$ by the ghost map

$$
w: W_{n}(\bar{V})^{\wedge} \rightarrow\left(\bar{V}^{\wedge}\right)^{n}
$$


is a $p^{m}$ th root of unity. Indeed, the ghost map is an injective ring homomorphism since the ring $\bar{V}^{\wedge}$ has no $p$-torsion. This, in turn, is equivalent to the statement that for all $0 \leq i<n$, the ghost coordinates

$$
w_{i}=a_{0}^{p^{i}}+p a_{1}^{p^{i-1}}+\cdots+p^{i-1} a_{i-1}^{p}+p^{i} a_{i}
$$

are $p^{m}$ th roots of unity in $\bar{V}^{\wedge}$. We show by induction on $0 \leq i<n$ that $a_{0}$ is a $p^{m}$ th root of unity in $\bar{V}^{\wedge}$ and that $a_{1}, \ldots, a_{i}$ are equal to zero. The statement for $i=0$ is trivial since $a_{0}=w_{0}$. So we assume that $a_{0}$ is a $p^{m}$ th root of unity and that $a_{1}=\cdots=a_{i-1}=0$ and must show that also $a_{i}=0$. The defining equation for the $i$ th ghost coordinate yields the equation

$$
w_{i}-a_{0}^{p^{i}}=p^{i} a_{i}
$$

Since $w_{i}$ and $a_{0}$ are both $p^{m}$ th roots of unity, the left-hand side is either zero or an element of valuation at most $1 /(p-1)$. Similarly, the right-hand side is either zero or an element of valuation at least $i$. It follows that $a_{i}=0$ as desired.

REMARK 1.3.5. In the proof of Lemma 1.3.4 we have used that $p$ is an odd prime. If $p=2$, the $2^{m}$ th roots of unity in $W_{n}(\bar{V})^{\wedge}$ are instead the elements of the form $\pm[\zeta]_{n}$ where $\zeta$ is a $2^{m}$ th root of unity in $\bar{V}^{\wedge}$. Hence, the number of $2^{m}$ th roots of unity in $W_{n}(\bar{V})^{\wedge}$ is equal to $2^{m}$, if $n=1$, and $2^{m+1}$, if $n>1$.

\section{The algebraically closed case}

2.1. In this section we determine the structure of the groups

$$
\operatorname{TR}_{q}^{n}\left(\bar{V} \mid \bar{K} ; p, \mathbb{Z}_{p}\right)=\pi_{q}\left(T(\bar{V} \mid \bar{K})^{C_{p^{n-1}}}, \mathbb{Z}_{p}\right)
$$

and prove Thms. $\mathrm{A}$ and $\mathrm{B}$ of the introduction. We first extend some of the results from $[8$ to the algebraically closed case.

The $p$-completion and $p$-cocompletion of a spectrum $X$ are defined by

$$
\begin{aligned}
& X^{\wedge}=F\left(M\left(\mathbb{Q}_{p} / \mathbb{Z}_{p},-1\right), X\right) \\
& X^{\llcorner}=M\left(\mathbb{Q}_{p} / \mathbb{Z}_{p},-1\right) \wedge X
\end{aligned}
$$

respectively [3. Here $M\left(\mathbb{Q}_{p} / \mathbb{Z}_{p},-1\right)$ is a Moore spectrum for the group $\mathbb{Q}_{p} / \mathbb{Z}_{p}$ concentrated in degree -1 . We write $\pi_{q}\left(X, \mathbb{Z}_{p}\right)$ and $\pi_{q}\left(X, \mathbb{Q}_{p} / \mathbb{Z}_{p}\right)$ for the $q$ th homotopy group of $X^{\wedge}$ and $X^{\varkappa}$, respectively. There are natural short-exact sequences

$$
\begin{aligned}
& 0 \rightarrow \operatorname{Ext}\left(\mathbb{Q}_{p} / \mathbb{Z}_{p}, \pi_{s} X\right) \rightarrow \pi_{s}\left(X, \mathbb{Z}_{p}\right) \rightarrow \operatorname{Hom}\left(\mathbb{Q}_{p} / \mathbb{Z}_{p}, \pi_{s-1} X\right) \rightarrow 0 \\
& 0 \rightarrow \pi_{s+1} X \otimes \mathbb{Q}_{p} / \mathbb{Z}_{p} \rightarrow \pi_{s}\left(X, \mathbb{Q}_{p} / \mathbb{Z}_{p}\right) \rightarrow \operatorname{Tor}\left(\pi_{s} X, \mathbb{Q}_{p} / \mathbb{Z}_{p}\right) \rightarrow 0
\end{aligned}
$$

which relate the integral homotopy groups and the homotopy groups with $\mathbb{Z}_{p^{-}}$ coefficients and with $\mathbb{Q}_{p} / \mathbb{Z}_{p}$-coefficients. A map of spectra induces a weak equivalence after $p$-completion if and only if it induces an isomorphism of homotopy groups with $\mathbb{Z} / p$-coefficients.

Let $\mathbb{T}$ be the circle group and let $C_{r}$ be the subgroup of order $r$. We begin with the following lemma from equivariant homotopy theory. We refer to [8, Sect. 4] for the definition and properties of the Tate spectrum.

LemMA 2.1.1. Let $X$ be a $\mathbb{T}$-spectrum and suppose the $\pi_{t} X$ vanishes for $t \ll 0$. Then the canonical map of Tate spectra

$$
\hat{\mathbb{H}}(\mathbb{T}, X) \rightarrow \underset{n}{\operatorname{holim}} \hat{\mathbb{H}}\left(C_{p^{n}}, X\right)
$$

becomes a weak equivalence after p-completion. 
Proof. We show first that the map

$$
\left(F\left(E_{+}, X\right)^{\mathbb{T}}\right)^{\wedge} \rightarrow \underset{n}{\operatorname{holim}}\left(F\left(E_{+}, X\right)^{C_{p^{n}}}\right)^{\wedge}
$$

is a weak equivalence. This can be rewritten as

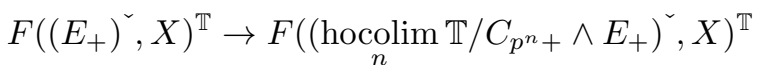

with the map induced from the canonical map

$$
\left.\underset{n}{\operatorname{hocolim}} \mathbb{T} / C_{p^{n}} \wedge E_{+}\right)^{\swarrow} \rightarrow\left(E_{+}\right)^{\swarrow} .
$$

This is a weak equivalence of pointed $\mathbb{T}$-spaces if and only if the map

$$
\left.\underset{n}{\operatorname{hocolim}} \mathbb{T} / C_{p^{n}+}\right)^{\iota} \rightarrow\left(S^{0}\right)^{\iota}
$$

is a weak equivalence of pointed spaces. The latter is easily verified by calculating homotopy groups.

We next show by induction that for $s \geq 0$, the canonical map

$$
\left(\left(\tilde{E}_{s} \wedge F\left(E_{+}, X\right)\right)^{\mathbb{T}}\right)^{\wedge} \rightarrow\left(\underset{n}{\operatorname{holim}}\left(\tilde{E}_{s} \wedge F\left(E_{+}, X\right)\right)^{C_{p^{n}}}\right)^{\wedge}
$$

is a weak equivalence. The basic case $s=0$ was proved above. To prove the induction step we consider the cofibration sequence

$$
\tilde{E}_{s-1} \longmapsto \tilde{E}_{s} \rightarrow \Sigma^{2 s} \mathbb{T}_{+} \rightarrow \Sigma \tilde{E}_{s-1} .
$$

It shows that it suffices to show that the map

$$
\left(\left(\Sigma^{2 s} \mathbb{T}_{+} \wedge F\left(E_{+}, X\right)\right)^{\mathbb{T}}\right)^{\wedge} \rightarrow\left(\underset{n}{\operatorname{holim}}\left(\Sigma^{2 s} \mathbb{T}_{+} \wedge F\left(E_{+}, X\right)\right)^{C_{p^{n}}}\right)^{\wedge}
$$

is a weak equivalence. Since the inclusion $X \rightarrow F\left(E_{+}, X\right)$ is a non-equivariant equivalence, we may instead show that the map

$$
\left(\left(\Sigma^{2 s} \mathbb{T}_{+} \wedge X\right)^{\mathbb{T}}\right)^{\wedge} \rightarrow\left(\underset{n}{\operatorname{holim}}\left(\Sigma^{2 s} \mathbb{T}_{+} \wedge X\right)^{C_{p^{n}}}\right)^{\wedge}
$$

is a weak equivalence. We now use the weak equivalence of $\mathbb{T}$-spectra

$$
\Sigma F\left(\mathbb{T}_{+}, X\right) \stackrel{\sim}{\rightarrow} \mathbb{T}_{+} \wedge X
$$

to rewrite the latter map as

$$
\left(\Sigma^{2 s+1} F\left(\mathbb{T}_{+}, X\right)^{\mathbb{T}}\right)^{\wedge} \rightarrow\left(\underset{n}{\operatorname{holim}}\left(\Sigma^{2 s+1} F\left(\mathbb{T}_{+}, X\right)^{C_{p^{n}}}\right)^{\wedge} .\right.
$$

As in the basic case $s=0$, we can rewrite this map as the map

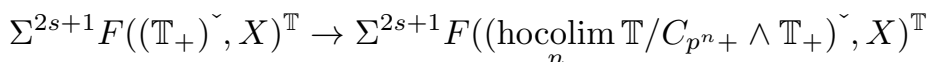

induced from the canonical map

$$
\left.\underset{n}{\operatorname{hocolim}} \mathbb{T} / C_{p^{n}} \wedge \mathbb{T}_{+}\right)^{\iota} \rightarrow\left(\mathbb{T}_{+}\right)^{\iota} .
$$

We see that this is a weak equivalence of pointed $\mathbb{T}$-spaces by calculating homotopy groups as before.

Finally, we consider the diagram

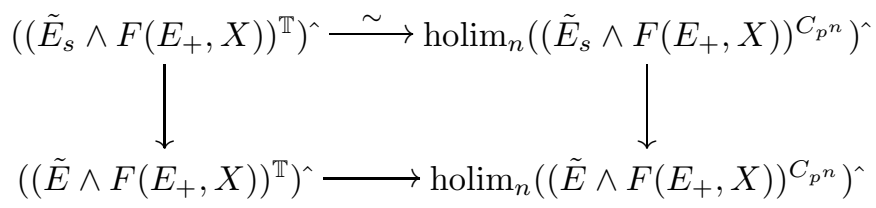


where the lower horizontal map is the $p$-completion of the map of the statement. The top horizontal map is an equivalence by what was shown above. Moreover, it follows from the proof of the induction step above that the map of $i$ th homotopy groups induced by each of vertical maps become isomorphisms for $s$ sufficiently large. This uses that $\pi_{t} X$ vanishes for $t \ll 0$. This completes the proof.

Let $G$ be a finite group, and let $T$ be a $G$-spectrum. There is a natural whole plane spectral sequence called the Tate spectral sequence

$$
\hat{E}_{s, t}^{2}(G, T)=\hat{H}^{-s}\left(G, \pi_{t}(T)\right) \Rightarrow \pi_{s+t}(\hat{\mathbb{H}}(G, T))
$$

that converges conditionally in the sense of [2 Def. 5.10] to the homotopy groups of the Tate spectrum and whose $E^{2}$-term is given by the Tate cohomology of $G$ with coefficients in the homotopy groups of $T$ considered as $G$-modules. The spectral sequence was first constructed in this generality in [5], but see also [8 Sect. 4].

LEMMA 2.1.3. Let $G$ be a finite group and let $\left\{T_{\alpha}\right\}$ be a filtrered colimit system of $G$-spectra. Suppose that for all $i \in \mathbb{Z}$, there exists constants $r$ and $c$ (independent of $\alpha$ ) such that $\hat{E}_{s, i-s}^{r}\left(G, T_{\alpha}\right)=\hat{E}_{s, i-s}^{\infty}\left(G, T_{\alpha}\right)$, for all $s \in \mathbb{Z}$, and such that $\hat{E}_{s, i-s}^{r}\left(G, T_{\alpha}\right)$ vanishes if $s<c$. Then the canonical map

$$
\operatorname{hocolim} \hat{\mathbb{H}}\left(G, T_{\alpha}\right) \rightarrow \hat{\mathbb{H}}\left(G, \operatorname{hocolim} T_{\alpha}\right)
$$

is a weak equivalence of spectra.

Proof. Let $\operatorname{Fil}_{s} \hat{\mathbb{H}}\left(G, T_{\alpha}\right)$, where $s$ ranges over the integers, be the filtration of $\hat{H}\left(G, T_{\alpha}\right)$ from $[8$. Sect. 4] which gives rise to the Tate spectral sequence (2.1.2). It induces a filtration of $\operatorname{hocolim}_{\alpha} \hat{\mathbb{H}}\left(G, T_{\alpha}\right)$, and the canonical map

$$
\underset{\alpha}{\operatorname{hocolim} \hat{\mathbb{H}}}\left(G, T_{\alpha}\right) \rightarrow \hat{\mathbb{H}}\left(G, \underset{\alpha}{\operatorname{hocolim}} T_{\alpha}\right)
$$

is filtration preserving. The filtration of the right-hand term gives rise to the spectral sequence $\hat{E}^{*}\left(G\right.$, hocolim $\left.T_{\alpha}\right)$. The filtration of the left-hand term gives rise to the colimit of the spectral sequences $\hat{E}^{*}\left(G, T_{\alpha}\right)$. Since Tate cohomology preserves filtered colimits, the map of spectral sequences induced by the map of the statement is an isomorphism. Hence we are done once we prove that both spectral sequences converge strongly. This is the case for each of the sequences $\hat{E}^{*}\left(G, T_{\alpha}\right)$. Indeed, this follows from [2 Thm. 8.2] since the sequences are conditionally convergent and collapse at the $E^{r}$-term. Since this is true for all $\alpha$, the spectral sequence $\hat{E}^{*}\left(G\right.$, hocolim $\left._{\alpha} T_{\alpha}\right)$ also converges strongly. We must show that the sequence

$$
\underset{\alpha}{\operatorname{colim}} \hat{E}^{*}\left(G, T_{\alpha}\right) \Rightarrow \pi_{*} \underset{\alpha}{\operatorname{hocolim}} \hat{\mathbb{H}}\left(G, T_{\alpha}\right)
$$

converges strongly. It suffices by loc. cit. to show that the spectral sequence converges conditionally which, by definition, means that

$$
\lim _{s} \operatorname{colim}_{\alpha} \pi_{i} \operatorname{Fil}_{s} \hat{\mathbb{H}}\left(G, T_{\alpha}\right)=R^{1} \lim _{s} \operatorname{colim}_{\alpha} \pi_{i+1} \operatorname{Fil}_{s} \hat{\mathbb{H}}\left(G, T_{\alpha}\right)=0 .
$$

But the assumptions imply that $\pi_{i} \operatorname{Fil}_{s} \hat{\mathbb{H}}\left(G, T_{\alpha}\right)$ vanishes for $s<c$ and for all $\alpha$. The lemma follows.

We can now prove the following generalization of [8, Addendum 5.4.4]. 
Proposition 2.1.4. Let $k$ be a perfect field of odd characteristic $p$, and let $K_{0}$ be the quotient field of the ring of Witt vectors $W(k)$. Let $K$ be an algebraic extension of $K_{0}$ and let $V$ be the integral closure of $W(k)$ in $K$. Then for all $n, v \geq 1$ and all $q \geq 0$, the map

$$
\hat{\Gamma}: \pi_{q}\left(\mathrm{TR}^{n}(V \mid K ; p), \mathbb{Z} / p^{v}\right) \rightarrow \pi_{q}\left(\hat{\mathbb{H}}\left(C_{p^{n}}, T(V \mid K)\right), \mathbb{Z} / p^{v}\right)
$$

is an isomorphism.

Proof. The statement was proved for finite field extensions in loc. cit. We write $K=\operatorname{colim}_{\alpha} K_{\alpha}$ as the filtered colimit of all finite subextensions of $K_{0}$ contained in $K$. Let $V_{\alpha}$ be the integral closure of $W(k)$ in $K_{\alpha}$. The colimit of the $V_{\alpha}$ maps isomorphically onto $V$, and hence, the induced map of $\mathbb{T}$-spectra

$$
\underset{\alpha}{\operatorname{hocolim}} T\left(V_{\alpha} \mid K_{\alpha}\right) \rightarrow T(V \mid K)
$$

induces an isomorphism on homotopy groups. We claim that also the induced map of $C_{p^{n-1} \text {-fixed point spectra }}$

$$
\underset{\alpha}{\operatorname{hocolim}} \operatorname{TR}^{n}\left(V_{\alpha} \mid K_{\alpha} ; p\right) \rightarrow \mathrm{TR}^{n}(V \mid K ; p)
$$

induces an isomorphism of homotopy groups. To prove the claim, we recall that by [7. Thm. 2.2], there is a cofibration sequence of spectra

$$
\mathbb{H} .\left(C_{p^{n-1}}, T(V \mid K)\right) \stackrel{N}{\rightarrow} \mathrm{TR}^{n}(V \mid K ; p) \stackrel{R}{\rightarrow} \mathrm{TR}^{n-1}(V \mid K ; p)
$$

that we call the fundamental cofibration sequence. The left-hand term is the Borel spectrum whose homotopy groups are the abutment of a natural strongly convergent first-quadrant spectral sequence

$$
E_{s, t}^{2}\left(C_{p^{n-1}}, T(V \mid K)\right)=H_{s}\left(C_{p^{n-1}}, \pi_{t}(T(V \mid K))\right) \Rightarrow \pi_{s+t}\left(\mathbb{H} .\left(C_{p^{n-1}}, T(V \mid K)\right)\right) .
$$

It follows that the induced map

$$
\underset{\alpha}{\operatorname{hocolim}} \mathbb{H} .\left(C_{p^{n}}, T\left(V_{\alpha} \mid K_{\alpha}\right)\right) \rightarrow \mathbb{H} .\left(C_{p^{n}}, \underset{\alpha}{\operatorname{hocolim}} T\left(V_{\alpha} \mid K_{\alpha}\right)\right)
$$

induces an isomorphism of homotopy groups. The claim then follows by easy induction over $n \geq 1$.

Finally, we use Lemma 2.1.3 to show that the canonical map

$$
\underset{\alpha}{\operatorname{hocolim}} \hat{\mathbb{H}}\left(C_{p^{n}}, T\left(V_{\alpha} \mid K_{\alpha}\right)\right) \rightarrow \hat{\mathbb{H}}\left(C_{p^{n}}, \underset{\alpha}{\operatorname{hocolim}} T\left(V_{\alpha} \mid K_{\alpha}\right)\right)
$$

induces an isomorphism of homotopy groups with $\mathbb{Z} / p^{v}$-coefficients. It suffices by an easy induction argument to consider the case $v=1$. Since the Moore spectrum $M_{p}$ for $\mathbb{Z} / p$ is a finite spectrum, the canonical map

$$
M_{p} \wedge \hat{\mathbb{H}}\left(C_{p^{n}}, T\left(V_{\alpha} \mid K_{\alpha}\right)\right) \rightarrow \hat{\mathbb{H}}\left(C_{p^{n}}, M_{p} \wedge T\left(V_{\alpha} \mid K_{\alpha}\right)\right)
$$

is a weak equivalence. Hence, it suffices to verify the hypotheses of Lemma 2.1.3 for the colimit system of $C_{p^{n} \text {-spectra }}\left\{T_{\alpha}\right\}$, where $T_{\alpha}=M_{p} \wedge T\left(V_{\alpha} \mid K_{\alpha}\right)$. It suffices as in the proof of [8, Thm. 5.4.3] to consider the case where $K_{\alpha}$ contains the $p$ th roots of unity. The differential structure of the spectral sequences

$$
\hat{E}_{s, t}^{2}\left(C_{p^{n}}, T_{\alpha}\right)=\hat{H}^{-s}\left(C_{p^{n}}, \pi_{t}\left(T_{\alpha}\right)\right) \Rightarrow \pi_{s+t}\left(\hat{\mathbb{H}}\left(C_{p^{n}}, T_{\alpha}\right)\right)
$$

was determined completely in op. cit., Thm. 5.5.1. In particular, it was shown there that for all $\alpha$ and for all $r \geq 2\left(p^{n+1}-1\right) /(p-1)$,

$$
\hat{E}^{r}\left(C_{p^{n}}, T_{\alpha}\right)=\hat{E}^{\infty}\left(C_{p^{n}}, T_{\alpha}\right) .
$$


This verifies the first of the two hypotheses of Lemma 2.1.3. The structure of the bi-graded abelian group $\hat{E}^{\infty}\left(C_{p^{n}}, T_{\alpha}\right)$ was determined in op. cit., Lemma 5.5.3. It follows, in particular, that for all $\alpha$ and for all $t \geq 2\left(p^{n+1}-1\right) /(p-1)-1$,

$$
\hat{E}_{s, t}^{\infty}\left(C_{p^{n}}, T_{\alpha}\right)=0 .
$$

This verfies the second hypothesis of Lemma 2.1.3 and hence the proposition.

2.2. It follows from $\left[\right.$ Thm. 3.3.8] that $\operatorname{TR}_{q}^{n}(\bar{V} \mid \bar{K} ; p)$ is zero, if $q$ is negative, and that the canonical map of Witt complexes

$$
W_{n} \Omega_{(\bar{V}, \bar{M})}^{q} \rightarrow \mathrm{TR}_{q}^{n}(\bar{V} \mid \bar{K} ; p)
$$

is an isomorphism in degrees $q \leq 2$. Indeed, both sides preserve filtered colimits. The following result concerns the structure of the de Rham-Witt groups on the left-hand side. The definition of the $W_{n}(\bar{V})$-module ${ }_{h} W_{n} \Omega_{(\bar{V}, \bar{M})}^{q}$ is given in op. cit., Sect. 3.2 .

Proposition 2.2.1. The group $W_{n} \Omega_{(\bar{V}, \bar{M})}^{q}$ is divisible, for all $q \geq 1$, and uniquely divisible, for all $q \geq 2$. Moreover, for all $q \geq 0$, the sequence is exact:

$$
0 \rightarrow{ }_{h} W_{n} \Omega_{(\bar{V}, \bar{M})}^{q} \stackrel{N}{\rightarrow} W_{n} \Omega_{(\bar{V}, \bar{M})}^{q} \stackrel{R}{\rightarrow} W_{n-1} \Omega_{(\bar{V}, \bar{M})}^{q} \rightarrow 0 .
$$

Proof. We first show that the sequence of the statement is exact. It suffices by [8. Prop. 3.2.6] to show that the left-hand map is injective. For $q \leq 1$, this follows from the proof of op. cit., Thm. 3.3.8. For $q \geq 2$, we claim that the canonical map

$$
\iota_{2}: F_{*}^{n-1} \Omega_{(\bar{V}, \bar{M})}^{q} \rightarrow{ }_{h} W_{n} \Omega_{(\bar{V}, \bar{M})}^{q}
$$

is an isomorphism and that the common group is uniquely divisible. The injectivity of the map $N$ follows since the composite

$$
F_{*}^{n-1} \Omega_{(\bar{V}, \bar{M})}^{q} \stackrel{\iota_{2}}{\longrightarrow}{ }_{h} W_{n} \Omega_{(\bar{V}, \bar{M})}^{q} \stackrel{N}{\longrightarrow} W_{n} \Omega_{(\bar{V}, \bar{M})}^{q} \stackrel{F^{n-1}}{\longrightarrow} F_{*}^{n-1} \Omega_{(\bar{V}, \bar{M})}^{q}
$$

is given by multiplication by $p^{n-1}$. To prove the claim, it suffices to show that the group $\Omega_{(\bar{V}, \bar{M})}^{q}$ is divisible, for $q \geq 1$, and uniquely divisible, for $q \geq 2$. Indeed, this follows immediately from op. cit., Lemma 3.2.5. We write $\bar{K}=\operatorname{colim}_{\alpha} K_{\alpha}$ as the filtered colimit of all finite sub-extension $K_{\alpha}$ of $K$ contained in $\bar{K}$. Then $\bar{V}=\operatorname{colim}_{\alpha} V_{\alpha}$, where $V_{\alpha}$ is the integral closure of $V$ in $K_{\alpha}$, and the canonical map

$$
\underset{\alpha}{\operatorname{colim}} \Omega_{\left(V_{\alpha}, M_{\alpha}\right)}^{q} \rightarrow \Omega_{(\bar{V}, \bar{M})}^{q}
$$

is an isomorphism. It follows from op. cit., Lemmas 2.2.3 and 2.2.4 that the common group is divisible, for $q \geq 1$, and uniquely divisible, for $q \geq 2$, as desired. This completes the proof that the sequence of the statement is exact.

Since the group ${ }_{h} W_{n} \Omega_{(\bar{V}, \bar{M})}^{q}$ is uniquely divisible, for $q \geq 2$, an easy induction argument based on the exact sequence of the statement shows that the group $W_{n} \Omega_{(\bar{V}, \bar{M})}^{q}$ is uniquely divisible, for $q \geq 2$, as stated. We will prove in Prop. 2.2.3 below that the group $W_{n} \Omega_{(\bar{V}, \bar{M})}^{1}$ is divisible.

We next consider the integral homotopy groups

$$
\mathrm{TH}_{q}(\bar{V} \mid \bar{K})=\mathrm{TR}_{q}^{1}(\bar{V} \mid \bar{K} ; p)
$$

of the topological Hochschild spectrum $T(\bar{V} \mid \bar{K})$. 
Lemma 2.2.2. The group $\mathrm{TH}_{q}(\bar{V} \mid \bar{K})$ is divisible, for all $q>0$, and uniquely divisible, for $q>0$ even. Moreover, for all integers $q$, the canonical map

$$
\Omega_{(\bar{V}, \bar{M})}^{q} \rightarrow \mathrm{TH}_{q}(\bar{V} \mid \bar{K})
$$

is a rational isomorophism.

Proof. We write $\bar{V}=\operatorname{colim}_{\alpha} V_{\alpha}$ as the filtered colimit of the integral closure of $V$ in the finite sub-extensions $K_{\alpha}$ of $K$ contained in $\bar{K}$. Then the canonical map

$$
\underset{\alpha}{\operatorname{colim}} \mathrm{TH}_{q}\left(V_{\alpha} \mid K_{\alpha}\right) \rightarrow \mathrm{TH}_{q}(\bar{V} \mid \bar{K})
$$

is an isomorphism. We recall from [8, Rem. 2.4.2] that for $q$ positive and even, the groups $\mathrm{TH}_{q}\left(V_{\alpha} \mid K_{\alpha}\right)$ are uniquely divisible, and from op. cit., Prop. 2.3.4, that for all integers $q$, the canonical map

$$
\Omega_{\left(V_{\alpha}, M_{\alpha}\right)}^{q} \rightarrow \mathrm{TH}_{q}\left(V_{\alpha} \mid K_{\alpha}\right)
$$

is a rational isomorphism. Hence the same holds for the groups $\operatorname{TH}_{q}(\bar{V} \mid \bar{K})$. It remains to show that for $q$ positive and odd, the group $\mathrm{TH}_{q}(\bar{V} \mid \bar{K})$ is divisible. To this end, we consider the homotopy groups with $\mathbb{Z} / p$-coefficients

$$
\mathrm{TH}_{q}(\bar{V} \mid \bar{K}, \mathbb{Z} / p)=\pi_{q}(T(\bar{V} \mid \bar{K}), \mathbb{Z} / p)
$$

It follows from op. cit., Thm. B and from the isomorphism at the beginning of the proof, that there is a canonical isomorphism of log-differential graded rings

$$
\Omega_{(\bar{V}, \bar{M})}^{*} \otimes_{\mathbb{Z}} S_{\mathbb{Z} / p}\{\kappa\} \stackrel{\sim}{\rightarrow} \mathrm{TH}_{*}(\bar{V} \mid \bar{K}, \mathbb{Z} / p) .
$$

But, as we saw in the proof of Prop. 2.2.1 above, $\Omega_{(\bar{V}, \bar{M})}^{1}$ is a divisible group, and hence this isomorphism becomes

$$
S_{\bar{V} / p \bar{V}}\{\kappa\} \stackrel{\sim}{\rightarrow} \mathrm{TH}_{*}(\bar{V} \mid \bar{K}, \mathbb{Z} / p)
$$

In particular, the homotopy groups with $\mathbb{Z} / p$-coefficients are concentrated in even degrees. It follows that for $q$ odd, the middle group in the short-exact sequence

$$
0 \rightarrow \mathrm{TH}_{q}(\bar{V} \mid \bar{K}) / p \rightarrow \mathrm{TH}_{q}(\bar{V} \mid \bar{K}, \mathbb{Z} / p) \rightarrow \mathrm{TH}_{q-1}(\bar{V} \mid \bar{K})[p] \rightarrow 0
$$

vanishes, and therefore, $\mathrm{TH}_{q}(\bar{V} \mid \bar{K})$ is divisible as stated. Here $A[p]$ denotes the subgroup of elements of order $p$ in the abelian group $A$.

Proposition 2.2.3. The group $\operatorname{TR}_{q}^{n}(\bar{V} \mid \bar{K} ; p)$ is divisible, if $q \geq 1$, uniquely divisible, if $q \geq 2$ is even, and for all integers $q$, the canonical map

$$
W_{n} \Omega_{(\bar{V}, \bar{M})}^{q} \rightarrow \mathrm{TR}_{q}^{n}(\bar{V} \mid \bar{K} ; p)
$$

is a rational isomorphism. Moreover, the restriction map

$$
R: \operatorname{TR}_{q}^{n}(\bar{V} \mid \bar{K} ; p) \rightarrow \mathrm{TR}_{q}^{n-1}(\bar{V} \mid \bar{K} ; p)
$$

is surjective, for all integers $q$ and all $n \geq 2$.

Proof. We again use the fundamental cofibration sequence

$$
\mathbb{H} .\left(C_{p^{n-1}}, T(\bar{V} \mid \bar{K})\right) \stackrel{N}{\rightarrow} \mathrm{TR}^{n}(\bar{V} \mid \bar{K} ; p) \stackrel{R}{\rightarrow} \mathrm{TR}^{n-1}(\bar{V} \mid \bar{K} ; p)
$$

which we used above in the proof of Prop. 2.1.4 We shall here follow the notation from [8, Sect. 3.3] and write

$$
{ }_{h} \operatorname{TR}_{q}^{n}(\bar{V} \mid \bar{K} ; p)=\pi_{q}\left(\mathbb{H} .\left(C_{p^{n-1}}, T(\bar{V} \mid \bar{K})\right)\right) .
$$


The long-exact sequence

$$
\cdots \stackrel{\partial}{\rightarrow}{ }_{h} \mathrm{TR}_{q}^{n}(\bar{V} \mid \bar{K} ; p) \stackrel{N}{\rightarrow} \mathrm{TR}_{q}^{n}(\bar{V} \mid \bar{K} ; p) \stackrel{R}{\rightarrow} \operatorname{TR}_{q}^{n-1}(\bar{V} \mid \bar{K} ; p) \stackrel{\partial}{\rightarrow} \cdots
$$

and the spectral sequence

$$
E_{s, t}^{2}=H_{s}\left(C_{p^{n-1}}, F_{*}^{n-1} \mathrm{TH}_{t}(\bar{V} \mid \bar{K})\right) \Rightarrow{ }_{h} \mathrm{TR}_{s+t}^{n}(\bar{V} \mid \bar{K} ; p)
$$

are both sequences of $W_{n}(\bar{V})$-modules. We recall from op. cit., Lemma 3.3.3 that, in addition, there is a canonical map of $W_{n}(\bar{V})$-modules

$$
{ }_{h} W_{n} \Omega_{(\bar{V}, \bar{M})}^{q} \rightarrow{ }_{h} \mathrm{TR}_{q}^{n}(\bar{V} \mid \bar{K} ; p)
$$

which explains the choice of notation.

We first show that the edge-homomorphism of the spectral sequence above

$$
F_{*}^{n-1} \mathrm{TH}_{q}(\bar{V} \mid \bar{K}) \rightarrow{ }_{h} \mathrm{TR}_{q}^{n}(\bar{V} \mid \bar{K} ; p)
$$

is an isomorphism, if $q>0$ and even, and an injection whose cokernel is a $p$ torsion group of bounded exponent, if $q>0$ and odd. Since the $C_{p^{n-1}}$-action on $T(\bar{V} \mid \bar{K})$ extends to an action by the full circle group $\mathbb{T}$, the induced $C_{p^{n-1} \text {-action }}$ on homotopy groups is trivial. Hence, Lemma 2.2.2 shows that $E_{s, t}^{2}$ is zero, if $s>0$ and $s+t$ is even, and a $p$-torsion group of bounded exponent, if $s>0$ and $s+t$ is odd. For degree reasons, the only possible non-zero differentials are

$$
d^{r}: E_{r, q-(r-1)}^{r} \rightarrow E_{0, q}
$$

with $r \geq 2$ and $q$ even. But this is a map from a $p$-torsion group to a uniquely divisible group and therefore is zero. Hence, all differentials in the spectral sequence are zero, and the edge-homomorphism is as stated. It follows that ${ }_{h} \operatorname{TR}_{q}^{n}(\bar{V} \mid \bar{K} ; p)$ is a uniquely divisible group, if $q>0$ is even, and the sum of a uniquely divisible group and a torsion group, if $q>0$ is odd.

We next show that for all $q$, the canonical map

$$
{ }_{h} W_{n} \Omega_{(\bar{V}, \bar{M})}^{q} \rightarrow{ }_{h} \mathrm{TR}_{q}^{n}(\bar{V} \mid \bar{K} ; p)
$$

is a rational isomorphism. To this end, we consider the following diagram.

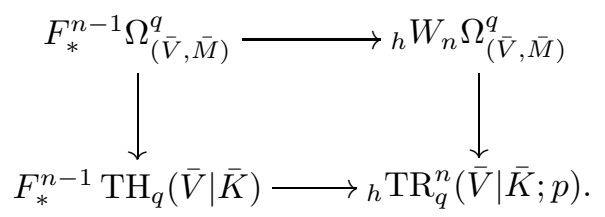

The lower horizontal map is the edge homomorphism of the spectral sequence and the remaining maps are the canonical ones. The top horizontal map is a rational isomorphism by the proof of Prop.2.2.1 and the left-hand vertical map is a rational isomorphism by Lemma 2.2.2 Finally, we proved above that the lower horizontal map is a rational isomorphism.

We proceed to show that the restriction map is surjective, or equivalently, that the long-exact sequence of homotopy groups associated with the fundamental cofibration sequence breaks into short-exact sequences

$$
0 \rightarrow{ }_{h} \mathrm{TR}_{q}^{n}(\bar{V} \mid \bar{K} ; p) \stackrel{N}{\rightarrow} \mathrm{TR}_{q}^{n}(\bar{V} \mid \bar{K} ; p) \stackrel{R}{\rightarrow} \mathrm{TR}_{q}^{n-1}(\bar{V} \mid \bar{K} ; p) \rightarrow 0 .
$$

It suffices to prove that this sequence is exact for $q$ even. Indeed, this implies that the boundary map in the long-exact sequence is zero, for all $q$. The sequence above 
is short-exact for $q=0$ by [7 Thm. F]. So we assume that $q \geq 2$ and even and consider the following diagram.

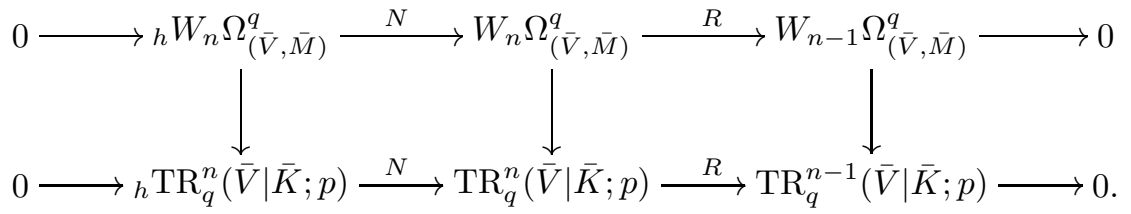

The top sequence is exact by Prop. 2.2.1 and we proved above that the left-hand vertical map is an isomorphism. Moreover, the lower left-hand map is injective. Indeed, the composite

$$
F_{*}^{n-1} \mathrm{TH}_{q}(\bar{V} \mid \bar{K}) \stackrel{\sim}{\longrightarrow}{ }_{h} \mathrm{TR}_{q}^{n}(\bar{V} \mid \bar{K} ; p) \stackrel{N}{\longrightarrow} \mathrm{TR}_{q}^{n}(\bar{V} \mid \bar{K} ; p) \stackrel{F^{n-1}}{\longrightarrow} F_{*}^{n-1} \mathrm{TH}_{q}(\bar{V} \mid \bar{K}),
$$

which is given by multiplication by $p^{n-1}$, is an isomorphism, and we proved above that the left-hand map is an isomorphism. It follows, by induction on $n \geq 1$, that the lower sequence is exact and that the middle vertical map is an isomorphism as desired.

It remains to prove that $\operatorname{TR}_{q}^{n}(\bar{V} \mid \bar{K} ; p)$ is divisible, if $q>0$ and odd. As in the proof of Lemma 2.2.2 we show that if $q>0$ and odd, the middle group in the following short-exact sequence vanishes.

$$
0 \rightarrow \mathrm{TR}_{q}^{n}(\bar{V} \mid \bar{K} ; p) / p \rightarrow \mathrm{TR}_{q}^{n}(\bar{V} \mid \bar{K} ; p, \mathbb{Z} / p) \rightarrow \mathrm{TR}_{q-1}^{n}(\bar{V} \mid \bar{K} ; p)[p] \rightarrow 0 .
$$

To prove this, we recall from Prop. 2.1.4 that for $q \geq 0$, the map

$$
\hat{\Gamma}: \operatorname{TR}_{q}^{n}(\bar{V} \mid \bar{K} ; p, \mathbb{Z} / p) \rightarrow \pi_{q}\left(\hat{\mathbb{H}}\left(C_{p^{n}}, T(\bar{V} \mid \bar{K})\right), \mathbb{Z} / p\right)
$$

is an isomorphism. We use the Tate spectral sequence

$$
\hat{E}_{s, t}^{2}\left(C_{p^{n}}\right)=\hat{H}^{-s}\left(C_{p^{n}}, \mathrm{TH}_{t}(\bar{V} \mid \bar{K}, \mathbb{Z} / p)\right) \Rightarrow \pi_{s+t}\left(\hat{\mathbb{H}}\left(C_{p^{n}}, \mathrm{TH}(\bar{V} \mid \bar{K})\right), \mathbb{Z} / p\right)
$$

to evaluate the right-hand side. As in the proof of Lemma 2.2.2 we find that

$$
\hat{E}^{2}\left(C_{p^{n}}\right)=\Lambda_{\bar{V} / p \bar{V}}\left\{u_{n}\right\} \otimes_{\bar{V} / p \bar{V}} S_{\bar{V} / p \bar{V}}\left\{t^{ \pm 1}, \kappa\right\}
$$

with the generators $u_{n}, t$, and $\kappa$ in bidegrees $(-1,0),(-2,0)$ and $(0,2)$, respectively. We recall from [8, Sect. 4.4] that there is a map to this spectral sequence from the spectral sequence

$$
\hat{E}^{2}(\mathbb{T})=S_{\bar{V} / p \bar{V}}\left\{t^{ \pm 1}, \kappa\right\} \Rightarrow \pi_{*}(\hat{\mathbb{H}}(\mathbb{T}, T(\bar{V} \mid \bar{K})), \mathbb{Z} / p)
$$

which on the $E^{2}$-terms is given by the obvious inclusion. For degree reasons, all differentials in the latter spectral sequence are zero. It follows that all possible non-zero differentials in the former spectral sequence are multiplicatively generated from a non-zero differential on the class $u_{n}$. Since $T(\bar{V} \mid \bar{K})$ is a $T\left(W(k) \mid K_{0}\right)$-module spectrum, [8. Prop. 5.5.4] shows that

$$
d^{2\left(\frac{p^{n+1}-1}{p-1}\right)-1} u_{n}=\mu_{n} \cdot(t \kappa)^{\frac{p^{n+1}-1}{p-1}-1} t,
$$

where $\mu_{n} \in \mathbb{Z} / p$ is a unit. We conclude that

$$
\hat{E}^{\infty}\left(C_{p^{n}}\right)=S_{\bar{V} / p \bar{V}}\left\{t^{ \pm 1}, \kappa\right\} /\left(\kappa^{\frac{p^{n+1}-1}{p-1}-1}\right),
$$

which is concentrated in even degrees as desired. 
2.3. We consider the homotopy groups with $\mathbb{Z}_{p}$-coefficients of $\operatorname{TR}^{n}(\bar{V} \mid \bar{K} ; p)$. We recall that for any spectrum $X$, the $p$-adic homotopy groups are related to the integral homotopy groups by a natural short-exact sequence

$$
0 \rightarrow \operatorname{Ext}\left(\mathbb{Q}_{p} / \mathbb{Z}_{p}, \pi_{q}(X)\right) \rightarrow \pi_{q}\left(X, \mathbb{Z}_{p}\right) \rightarrow \operatorname{Hom}\left(\mathbb{Q}_{p} / \mathbb{Z}_{p}, \pi_{q-1}(X)\right) \rightarrow 0 .
$$

The right-hand term is also written $T_{p}\left(\pi_{q-1}(X)\right)$ and called the $p$-primary Tate module of the group $\pi_{q-1}(X)$. In the case at hand, the left-hand term (resp. the right-hand term) vanishes for $q>0$ (resp. for $q>0$ and odd) since $\operatorname{TR}_{q}^{n}(\bar{V} \mid \bar{K} ; p)$ is divisible (resp. torsion-free). In particular, we obtain a canonical isomorphism

$$
\operatorname{TR}_{2}^{n}\left(\bar{V} \mid \bar{K} ; p, \mathbb{Z}_{p}\right) \stackrel{\sim}{\rightarrow} T_{p} \operatorname{TR}_{1}^{n}(\bar{V} \mid \bar{K} ; p) \stackrel{\sim}{\leftarrow} T_{p} W_{n} \Omega_{(\bar{V}, \bar{M})}^{1}
$$

which, in turn, gives rise to a map of graded $W_{n}(\bar{V})^{\wedge}$-algebras

$$
S_{W_{n}(\bar{V})^{\wedge}}\left(T_{p} W_{n} \Omega_{(\bar{V}, \bar{M})}^{1}\right) \rightarrow \mathrm{TR}_{*}^{n}\left(\bar{V} \mid \bar{K} ; p, \mathbb{Z}_{p}\right) .
$$

We first consider this map for $n=1$.

Lemma 2.3.1. The p-primary Tate module $T_{p} \Omega_{(\bar{V}, \bar{M})}^{1}$ is a free $\bar{V}^{\wedge}$-module of rank one and the canonical map

$$
S_{\bar{V}^{\wedge}}\left(T_{p} \Omega_{(\bar{V}, \bar{M})}^{1}\right) \rightarrow \mathrm{TH}_{*}\left(\bar{V} \mid \bar{K}, \mathbb{Z}_{p}\right)
$$

is an isomorphism.

Proof. We have a canonical isomorphism

$$
T_{p} \Omega_{(\bar{V}, \bar{M})}^{1} \stackrel{\sim}{\rightarrow} \lim _{m} \Omega_{(\bar{V}, \bar{M})}^{1}\left[p^{m}\right]
$$

of the $p$-primary Tate module and the limit over $m \geq 1$ of the $p^{m}$-torsion subgroups. Let $v^{(m)} \in \bar{V}, m \geq 1$, with $v^{(1)}=-p$ and $\left(v^{(m+1)}\right)^{p}=v^{(m)}$. Then

$$
\Omega_{(\bar{V}, \bar{M})}^{1}\left[p^{m}\right]=\bar{V} / p^{m} \bar{V} \cdot d \log v^{(m)} .
$$

Indeed, the case $m=1$ follows from [8, Cor. 2.2.5], and the general case is proved by induction using the following diagram.

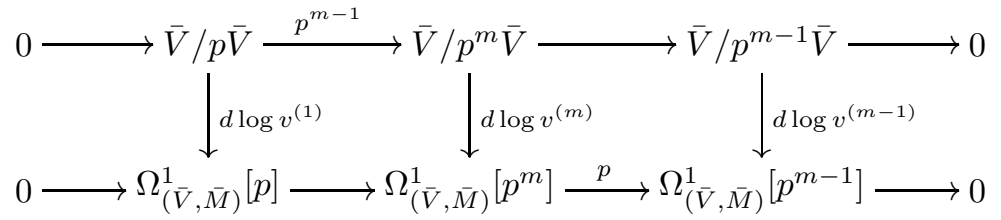

The lower horizontal sequence is canonically identified with the coefficients sequence

$$
0 \rightarrow \mathrm{TH}_{2}(\bar{V} \mid \bar{K}, \mathbb{Z} / p) \rightarrow \mathrm{TH}_{2}\left(\bar{V} \mid \bar{K}, \mathbb{Z} / p^{m}\right) \rightarrow \mathrm{TH}_{2}\left(\bar{V} \mid \bar{K}, \mathbb{Z} / p^{m-1}\right) \rightarrow 0
$$

which is exact since $\mathrm{TH}_{q}\left(\bar{V} \mid \bar{K}, \mathbb{Z} / p^{v}\right)$ is zero for $q$ odd. It follows that the $p$-primary Tate module $T_{p} \Omega_{(\bar{V}, \bar{M})}^{1}$ is a free $\bar{V}^{\wedge}$-module of rank one and that $\kappa=\left\{d \log v^{(m)}\right\}_{m \geq 1}$ is a generator. It remains to show that the composite map

$$
S^{2} \wedge \mathrm{TH}(\bar{V} \mid \bar{K})^{\wedge} \stackrel{\kappa \wedge \text { id }}{\longrightarrow} \mathrm{TH}(\bar{V} \mid \bar{K})^{\wedge} \wedge \mathrm{TH}(\bar{V} \mid \bar{K})^{\wedge} \stackrel{\mu}{\rightarrow} \mathrm{TH}(\bar{V} \mid \bar{K})^{\wedge}
$$

induces an isomorphism of homotopy groups in degrees $q \geq 2$. Since this is a map between $p$-complete spectra, it suffices to show that the induced map of homotopy groups with $\mathbb{Z} / p$-coefficients is an isomorphism in degrees $q \geq 2$. But this follows from [8, Thm. B]. 
The following result together with Prop. 2.2.3 above completes the proof of Thm. \$ of the induction.

Proposition 2.3.2. The p-primary Tate module $T_{p} W_{n} \Omega_{(\bar{V}, \bar{M})}^{1}$ is a free module of rank one over $W_{n}(\bar{V})^{\wedge}$ and the canonical map

$$
S_{W_{n}(\bar{V})^{\wedge}}\left(T_{p} W_{n} \Omega_{(\bar{V}, \bar{M})}^{1}\right) \rightarrow \mathrm{TR}_{*}^{n}\left(\bar{V} \mid \bar{K} ; p, \mathbb{Z}_{p}\right)
$$

is an isomorphism.

Proof. We know from Prop. 2.1.4 that the map

$$
\hat{\Gamma}: \operatorname{TR}^{n}(\bar{V} \mid \bar{K} ; p) \rightarrow \hat{\mathbb{H}}\left(C_{p^{n}}, \operatorname{TH}(\bar{V} \mid \bar{K})\right)
$$

induces an isomorphism of homotopy groups with $\mathbb{Z}_{p}$-coefficients in non-negative degrees. It follows from Lemma 2.3.1 that the Tate spectral sequence

$$
\hat{E}_{s, t}^{2}=\hat{H}^{-s}\left(C_{p^{n}}, \mathrm{TH}_{t}\left(\bar{V} \mid \bar{K}, \mathbb{Z}_{p}\right)\right) \Rightarrow \pi_{s+t}\left(\hat{\mathbb{H}}\left(C_{p^{n}}, \mathrm{TH}(\bar{V} \mid \bar{K})\right), \mathbb{Z}_{p}\right)
$$

takes the form $\hat{E}^{2}=S_{\bar{V} / p^{n} \bar{V}}\left\{t^{ \pm 1}, \kappa\right\}$ with $t$ and $\kappa$ in bi-degrees $(-2,0)$ and $(0,2)$, respectively. Since all non-zero elements are located in even total degree, all differentials are zero and the groups $\operatorname{TR}_{q}^{n}\left(\bar{V} \mid \bar{K} ; p, \mathbb{Z}_{p}\right)$ are concentrated in even degrees. Moreover, the spectral sequence is multiplicative, and multiplication by $t^{-1}$ induces an isomorphism of $\hat{E}_{s, t}^{\infty}$ onto $\hat{E}_{s+2, t}^{\infty}$. Let $\alpha_{n} \in \mathrm{TR}_{2}^{n}\left(\bar{V} \mid \bar{K} ; p, \mathbb{Z}_{p}\right)$ be a homotopy class such that $\hat{\Gamma}\left(\alpha_{n}\right)$ is represented in the spectral sequence by the element $t^{-1}$. Then multiplication by $\alpha_{n}$ induces an isomorphism

$$
\operatorname{TR}_{q}^{n}\left(\bar{V} \mid \bar{K} ; p, \mathbb{Z}_{p}\right) \stackrel{\sim}{\rightarrow} \operatorname{TR}_{q+2}^{n}\left(\bar{V} \mid \bar{K} ; p, \mathbb{Z}_{p}\right)
$$

for all positive integers $q$. This proves the proposition.

AdDendum 2.3.3. The Frobenius

$$
F: \operatorname{TR}_{q}^{n}\left(\bar{V} \mid \bar{K} ; p, \mathbb{Z}_{p}\right) \rightarrow \operatorname{TR}_{q}^{n-1}\left(\bar{V} \mid \bar{K} ; p, \mathbb{Z}_{p}\right)
$$

is surjective.

Proof. For $q=0$, the statement is that $F: W_{n}(\bar{V})^{\wedge} \rightarrow W_{n-1}(\bar{V})^{\wedge}$ is surjective which we proved in Cor. 1.2.2 Hence it suffices by Prop. 2.3.2 to show that

$$
F: \operatorname{TR}_{2}^{n}\left(\bar{V} \mid \bar{K} ; p, \mathbb{Z}_{p}\right) \rightarrow \mathrm{TR}_{2}^{n-1}\left(\bar{V} \mid \bar{K} ; p, \mathbb{Z}_{p}\right)
$$

takes a $W_{n}(\bar{V})^{\wedge}$-module generator to a $W_{n-1}(\bar{V})^{\wedge}$-module generator. By Prop. 2.1.4 we may instead show that the map

$$
F: \pi_{2}\left(\hat{\mathbb{H}}\left(C_{p^{n}}, T(\bar{V} \mid \bar{K})\right), \mathbb{Z}_{p}\right) \rightarrow \pi_{2}\left(\hat{\mathbb{H}}\left(C_{p^{n-1}}, T(\bar{V} \mid \bar{K})\right), \mathbb{Z}_{p}\right)
$$

takes a $W_{n}(\bar{V})^{\wedge}$-module generator to a $W_{n-1}(\bar{V})^{\wedge}$-module generator. It follows from the construction of the Tate spectral sequence that the latter map induces a map of Tate spectral sequences that on $E^{2}$-terms is given by the map

$$
S_{\bar{V} / p^{n} \bar{V}}\left\{t^{ \pm 1}, \kappa\right\} \rightarrow S_{\bar{V} / p^{n-1} \bar{V}}\left\{t^{ \pm 1}, \kappa\right\}
$$

that takes generators $t$ and $\kappa$ on the left-hand side to the generators $t$ and $\kappa$ on the right-hand side and that maps $\bar{V} / p^{n} \bar{V}$ to $\bar{V} / p^{n-1} \bar{V}$ by the canonical projection; see [8 Sect. 4]. The element $t^{-1}$ on the left-hand side represents a $W_{n}(\bar{V})^{\wedge}$-module generator of $\pi_{2}\left(\hat{\mathbb{H}}\left(C_{p^{n}}, T(\bar{V} \mid \bar{K})\right), \mathbb{Z}_{p}\right)$ and the element $t^{-1}$ on the right-hand side represents a $W_{n-1}(\bar{V})^{\wedge}$-module generator of $\pi_{2}\left(\hat{\mathbb{H}}\left(C_{p^{n-1}}, T(\bar{V} \mid \bar{K})\right), \mathbb{Z}_{p}\right)$. This completes the proof. 
We conclude this paragraph with a brief discussion of the homotopy groups with $\mathbb{Q}_{p} / \mathbb{Z}_{p}$-coefficients of the spectrum $\operatorname{TR}^{n}(\bar{V} \mid \bar{K} ; p)$.

LEMma 2.3.4. Let $A$ be a divisible abelian group and let $T_{p}(A)$ be the p-primary Tate module of $A$. Then there is a canonical isomorphism

$$
\operatorname{Tor}\left(A, \mathbb{Q}_{p} / \mathbb{Z}_{p}\right) \stackrel{\sim}{\rightarrow} T_{p}(A) \otimes \mathbb{Q}_{p} / \mathbb{Z}_{p} .
$$

Proof. Since $A$ is a divisible abelian group, the short-exact sequence

$$
0 \rightarrow \mathbb{Z} \rightarrow \mathbb{Z}\left[\frac{1}{p}\right] \rightarrow \mathbb{Q}_{p} / \mathbb{Z}_{p} \rightarrow 0
$$

induces a short-exact

$$
0 \rightarrow \operatorname{Hom}\left(\mathbb{Q}_{p} / \mathbb{Z}_{p}, A\right) \rightarrow \operatorname{Hom}\left(\mathbb{Z}\left[\frac{1}{p}\right], A\right) \rightarrow \operatorname{Hom}(\mathbb{Z}, A) \rightarrow 0
$$

that is usually written

$$
0 \rightarrow T_{p}(A) \rightarrow V_{p}(A) \rightarrow A \rightarrow 0 .
$$

This sequence, in turn, gives rise to a six-term exact sequence

$$
\begin{aligned}
0 & \rightarrow \operatorname{Tor}\left(T_{p}(A), \mathbb{Q}_{p} / \mathbb{Z}_{p}\right) \rightarrow \operatorname{Tor}\left(V_{p}(A), \mathbb{Q}_{p} / \mathbb{Z}_{p}\right) \rightarrow \operatorname{Tor}\left(A, \mathbb{Q}_{p} / \mathbb{Z}_{p}\right) \\
& \rightarrow T_{p}(A) \otimes \mathbb{Q}_{p} / \mathbb{Z}_{p} \rightarrow V_{p}(A) \otimes \mathbb{Q}_{p} / \mathbb{Z}_{p} \rightarrow A \otimes \mathbb{Q}_{p} / \mathbb{Z}_{p} \rightarrow 0 .
\end{aligned}
$$

To prove the statement of the lemma, it suffices to prove that the second and fifth terms of this sequence vanish. But this follows immediately from the exact sequence at the beginning of the proof considered as a flat resolution of $\mathbb{Q}_{p} / \mathbb{Z}_{p}$ and from the fact that $V_{p}(A)$ is a $\mathbb{Z}\left[\frac{1}{p}\right]$-module.

Corollary 2.3.5. For all integers $q$ and all positive integers $n$, there is a canonical isomorphism of abelian groups

$$
\operatorname{TR}_{q}^{n}\left(\bar{V} \mid \bar{K} ; p, \mathbb{Q}_{p} / \mathbb{Z}_{p}\right) \stackrel{\sim}{\rightarrow} \operatorname{TR}_{q}^{n}\left(\bar{V} \mid \bar{K} ; p, \mathbb{Z}_{p}\right) \otimes \mathbb{Q}_{p} / \mathbb{Z}_{p}
$$

Proof. This follows immediately from Thm. A Lemma 2.3.4 and from the definition of homotopy groups with $\mathbb{Q}_{p} / \mathbb{Z}_{p}$-coefficients which we recalled at the beginning of Sect. 2 above.

2.4. Let $\varepsilon=\left\{\varepsilon^{(v)}\right\}_{v \geq 1}$ be a sequence of primitive $p^{v-1}$ th roots of unity in $\bar{K}$ that are compatible in the sense that $\left(\varepsilon^{(v+1)}\right)^{p}=\varepsilon^{(v)}$. The sequence $\varepsilon$ is a generator of the $p$-primary Tate module $T_{p}\left(\bar{K}^{*}\right)$, and the image by the canonical isomorphism

$$
T_{p}\left(\bar{K}^{*}\right) \stackrel{\sim}{\rightarrow} T_{p} K_{1}(\bar{K})
$$

is the associated $K$-theory Bott element $\beta_{\varepsilon}=\beta_{\varepsilon}^{K}$.

Lemma 2.4.1. The image of the $K$-theory Bott element $\beta_{\varepsilon}^{K}$ by the map

$$
T_{p} K_{1}(\bar{K}) \rightarrow T_{p} \operatorname{TR}_{1}^{n}(\bar{V} \mid \bar{K} ; p)
$$

induced by the cyclotomic trace is the element $\beta_{\varepsilon, n}=\beta_{\varepsilon, n}^{\mathrm{TR}}=\left\{d \log _{n} \varepsilon^{(v)}\right\}_{v \geq 1}$.

Proof. We recall from [8 Sect. 2.3] that the map

$$
d \log _{n}: \bar{M}=\bar{V} \cap \bar{K}^{*} \rightarrow \mathrm{TR}_{1}^{n}(\bar{V} \mid \bar{K} ; p)
$$

is defined to be the composite of the canonical inclusion $\alpha: \bar{M} \hookrightarrow \bar{K}^{*}$, the canonical isomorphism $\bar{K}^{*} \rightarrow K_{1}(\bar{K})$, and the cyclotomic trace $K_{1}(\bar{K}) \rightarrow \mathrm{TR}_{1}^{n}(\bar{V} \mid \bar{K} ; p)$. The stated formula is now clear. 
The TR-theory Bott element

$$
\beta_{\varepsilon, n}=\beta_{\varepsilon, n}^{\mathrm{TR}} \in T_{p} \mathrm{TR}_{1}^{n}(\bar{V} \mid \bar{K} ; p) \stackrel{\sim}{\leftarrow} T_{p} W_{n} \Omega_{(\bar{V}, \bar{M})}^{1}
$$

satisfies that $R\left(\beta_{n, \varepsilon}\right)=F\left(\beta_{n, \varepsilon}\right)=\beta_{n-1, \varepsilon}$ and hence defines a Bott element

$$
\beta_{\varepsilon}=\beta_{\varepsilon}^{\mathrm{TF}}=\left\{\beta_{\varepsilon, n}\right\}_{n \geq 1} \in \lim _{F} T_{p} W_{n} \Omega_{(\bar{V}, \bar{M})}^{1} .
$$

The right-hand group is a free module of rank one over the $\operatorname{ring} \lim _{F} W_{n}(\bar{V})^{\wedge}$ which we identify with the ring $W\left(R_{\bar{V}}\right)$ via ring isomorphism

$$
\psi: W\left(R_{\bar{V}}\right) \stackrel{\sim}{\rightarrow} \lim _{F} W_{n}(\bar{V})^{\wedge}
$$

of Addendum 1.2.4 The Bott element $\beta_{\varepsilon}$, however, is not a generator. Instead we shall prove the following result. Let $\varepsilon_{n}=\left\{\varepsilon^{(v+n)}\right\}_{v \geq 1}$. We denote by $\varepsilon_{n}$ the corresponding element of the ring $R_{\bar{V}}$ and by $\left[\varepsilon_{n}\right]$ the associated Teichmüller representative of the Witt ring $W\left(R_{\bar{V}}\right)$.

Proposition 2.4.2. There exists a unique $W\left(R_{\bar{V}}\right)$-module generator

$$
\alpha_{\varepsilon} \in \lim _{F} T_{p} W_{n} \Omega_{(\bar{V}, \bar{M})}^{1}
$$

with the property that $\beta_{\varepsilon}=\psi\left(\left[\varepsilon_{1}\right]-1\right) \cdot \alpha_{\varepsilon}$.

The uniqueness part of Prop. 2.4 .2 follows immediately from Addendum 1.3.2 the existence part is proved in Sect. 3 below. We conclude this section with the proofs of Thm. B and $\mathbb{C}$ of the introduction.

Proof of Thm. B. We first prove the statement (i) of Thm. B It follows from Addendum 2.3.3 that the canonical projection

$$
\operatorname{pr}_{n, 1}: \lim _{F} T_{p} W_{n} \Omega_{(\bar{V}, \bar{M})}^{1} \rightarrow T_{p} W_{n} \Omega_{(\bar{V}, \bar{M})}^{1}
$$

is surjective. Similarly, Cor. 1.2 .2 shows that the canonical projection

$$
\operatorname{pr}_{n, 0}: \lim _{F} W_{n}(\bar{V})^{\wedge} \rightarrow W_{n}(\bar{V})^{\wedge}
$$

is surjective. Moreover, the domain (resp. the range) of the map $\mathrm{pr}_{n, 1}$ is a free module of rank one over the domain (resp. range) of the map $\mathrm{pr}_{n, 0}$. It follows that the map $\mathrm{pr}_{n, 1}$ takes a generator to a generator. Hence, the class defined by

$$
\alpha_{\varepsilon, n}=\operatorname{pr}_{n, 1}\left(\alpha_{\varepsilon}\right) \in T_{p} W_{n} \Omega_{(\bar{V}, \bar{M})}^{1}
$$

is a $W_{n}(\bar{V})^{\wedge}$-module generator. Finally,

$$
\begin{aligned}
\beta_{\varepsilon, n} & =\operatorname{pr}_{n, 1}\left(\beta_{\varepsilon}\right)=\operatorname{pr}_{n, 1}\left(\psi\left(\left[\varepsilon_{1}\right]-1\right) \cdot \alpha_{\varepsilon}\right) \\
& =\operatorname{pr}_{n, 0}\left(\psi\left(\left[\varepsilon_{1}\right]-1\right)\right) \cdot \operatorname{pr}_{n, 1}\left(\alpha_{\varepsilon}\right)=\theta_{n}\left(\left[\varepsilon_{n}\right]-1\right) \cdot \alpha_{\varepsilon, n}
\end{aligned}
$$

as stated.

We next consider the statement (ii) of Thm. B It is immediate from the definition of $\alpha_{\varepsilon, n}$ that $F\left(\alpha_{\varepsilon, n}\right)=\alpha_{\varepsilon, n-1}$. The restriction map induces a self-map

$$
R: \lim _{F} T_{p} W_{n} \Omega_{(\bar{V}, \bar{M})}^{1} \rightarrow \lim _{F} T_{p} W_{n} \Omega_{(\bar{V}, \bar{M})}^{1}
$$

of a free $W\left(R_{\bar{V}}\right)$-module of rank one. Hence there exists an element $\xi \in W\left(R_{\bar{V}}\right)$ such that $R\left(\alpha_{\varepsilon}\right)=\psi(\xi) \cdot \alpha_{\varepsilon}$, and since $W\left(R_{\bar{V}}\right)$ is an integral domain, this element $\xi$ is unique. Since $R\left(\beta_{\varepsilon}\right)=\beta_{\varepsilon}$ and $\beta_{\varepsilon}=\psi\left(\left[\varepsilon_{1}\right]-1\right) \cdot \alpha_{\varepsilon}$, we find that

$$
\psi\left(\left[\varepsilon_{1}\right]-1\right) \cdot \alpha_{\varepsilon}=R\left(\psi\left(\left[\varepsilon_{1}\right]-1\right) \cdot \alpha_{\varepsilon}\right)=\psi\left(\left[\varepsilon_{2}\right]-1\right) \cdot R\left(\alpha_{\varepsilon}\right)
$$


which shows that $\xi=\left(\left[\varepsilon_{1}\right]-1\right) /\left(\left[\varepsilon_{2}\right]-1\right)$. The stated formula now follows from Addendum 1.2.4

The proof of statement (iii) of Thm. $\mathbb{B}$ is similar to the proof of statement (ii) above. We have $\beta_{\varepsilon}^{\sigma}=\chi(\sigma) \cdot \beta_{\varepsilon}$ and $\beta_{\varepsilon}=\psi\left(\left[\varepsilon_{1}\right]-1\right) \cdot \alpha_{\varepsilon}$ which shows that

$$
\psi\left(\left[\varepsilon_{1}^{\sigma}\right]-1\right) \cdot \alpha_{\varepsilon}^{\sigma}=\chi(\sigma) \cdot \psi\left(\left[\varepsilon_{1}\right]-1\right) \cdot \alpha_{\varepsilon} .
$$

This is an equation among elements of a free module of rank one over an integral domain. Hence, we obtain

$$
\alpha_{\varepsilon}^{\sigma}=\chi(\sigma) \cdot \psi\left(\left(\left[\varepsilon_{1}\right]-1\right) /\left(\left[\varepsilon_{1}^{\sigma}\right]-1\right)\right) \cdot \alpha_{\varepsilon}
$$

and apply Addendum 1.2 .4 to obtain the stated formula.

Proof of Thm. [C] Let $\operatorname{TF}(\bar{V} \mid \bar{K} ; p)$ be the homotopy limit spectrum

$$
\mathrm{TF}(\bar{V} \mid \bar{K} ; p)=\underset{F}{\operatorname{holim}} \operatorname{TR}^{n}(\bar{V} \mid \bar{K} ; p)
$$

where the structure map in the limit system is the Frobenius map. The restriction and Frobenius maps give rise to self-maps of the spectrum $\operatorname{TF}(\bar{V} \mid \bar{K} ; p)$ and the topological cyclic homology spectrum $\operatorname{TC}(\bar{V} \mid \bar{K} ; p)$ is defined to be the homotopy equalizer. Hence, the homotopy groups form a long-exact sequence

$$
\ldots \rightarrow \mathrm{TC}_{q}(\bar{V} \mid \bar{K} ; p) \rightarrow \mathrm{TF}_{q}(\bar{V} \mid \bar{K} ; p) \stackrel{1-R}{\longrightarrow} \mathrm{TF}_{q}(\bar{V} \mid \bar{K} ; p) \stackrel{\partial}{\rightarrow} \mathrm{TC}_{q-1}(\bar{V} \mid \bar{K} ; p) \rightarrow \ldots
$$

and we have the Milnor short-exact sequence

$$
0 \rightarrow R^{1} \lim _{F} \operatorname{TR}_{q+1}^{n}(\bar{V} \mid \bar{K} ; p) \rightarrow \mathrm{TF}_{q}(\bar{V} \mid \bar{K} ; p) \rightarrow \lim _{F} \operatorname{TR}_{q}^{n}(\bar{V} \mid \bar{K} ; p) \rightarrow 0 .
$$

The same is true for the homotopy groups with $\mathbb{Z}_{p}$-coefficients. It follows from Thms. $\mathrm{A}$ and $\mathrm{B}$ that the map

$$
g_{\varepsilon, m}: W\left(R_{\bar{V}}\right) \rightarrow \mathrm{TF}_{2 m}\left(\bar{V} \mid \bar{K} ; p, \mathbb{Z}_{p}\right)
$$

that takes $a$ to $\psi(a) \cdot \alpha_{\varepsilon}^{m}$ is an isomorphism, for all non-negative integers $m$, and that the groups in odd degrees are zero. Moreover, the following diagram commutes.

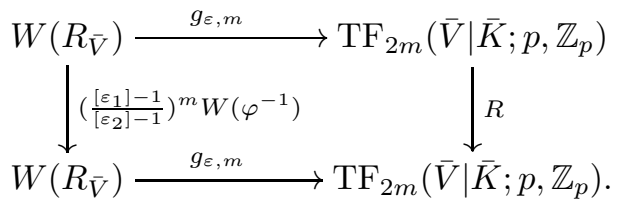

The statement of the theorem now follows from Cor. 1.3.3

\section{The generator $\alpha_{\varepsilon}$}

3.1. We obtain the generator $\alpha_{\varepsilon}$ from the Thom class $\lambda_{-L}$ in topological $K$ theory associated with negative the canonical line bundle over $\mathbb{P}^{\infty}(\mathbb{C})$. We first recall some standard facts about topological $K$-theory.

Let $k u$ be the connective cover of the $p$-completion of the spectrum representing periodic complex $K$-theory. This is a ring spectrum whose homotopy groups

$$
\pi_{*}(k u)=S_{\mathbb{Z}_{p}}\{\beta\}
$$

is the polynomial algebra over $\mathbb{Z}_{p}$ on the Bott element $\beta=\beta^{k u}$ of degree 2 .

The canonical projection $f: \mathbb{T} \rightarrow\{1\}$ from the circle group to the trivial group gives rise to an adjoint pair of functors $\left(f^{*}, f_{*}\right)$ between the stable homotopy category and the $\mathbb{T}$-stable homotopy category. (The functor $f_{*}$ was left out of the 
notation in the bottom row of the diagram at the beginning of the introduction.) We consider the Tate spectrum of the $\mathbb{T}$-spectrum $f^{*} k u$ obtained from the spectrum $k u$ in this way. The Tate spectral sequence takes the form

$$
\hat{E}^{2}=S_{\mathbb{Z}_{p}}\left\{t^{ \pm 1}, \beta\right\} \Rightarrow \pi_{*}\left(\hat{\mathbb{H}}\left(\mathbb{T}, f^{*} k u\right)\right)
$$

with the generators $t$ and $\beta$ located in bi-degrees $(-2,0)$ and $(0,2)$, respectively. Since the non-zero groups are all located in even total degrees, it follows that all differentials are zero. Hence, the graded $\operatorname{ring} \pi_{*}\left(\hat{\mathbb{H}}\left(\mathbb{T}, f^{*} k u\right)\right)$ is the tensor product of a power series algebra and a Laurent polynomial algebra over $\mathbb{Z}_{p}$ on generators $h$ and $\lambda_{-L}$ that represent $t \beta$ and $t^{-1}$, respectively. We now explicitly choose a pair of generators $h$ and $\lambda_{-L}$.

Let $L=\mathcal{O}(1)$ be the canonical line bundle over $\mathbb{P}^{\infty}(\mathbb{C})$ and define $\mathbb{P}_{-s}^{\infty}(\mathbb{C})$ to be the Thom spectrum of the virtual bundle $-s L$. We recall that $\mathbb{P}_{-s}^{\infty}(\mathbb{C})$ may be given the structure of a CW-spectrum with one cell in every even dimension greater than or equal to $-2 s$. Moreover, it follows from [10. Prop. II.4.4] that for all integers $s$, there is a canonical isomorphism

$$
\pi_{2 s}\left(\hat{\mathbb{H}}\left(\mathbb{T}, f^{*} k u\right)\right) \stackrel{\sim}{\longrightarrow} K^{-2 s}\left(\mathbb{P}_{-s}^{\infty}(\mathbb{C}), \mathbb{Z}_{p}\right) .
$$

The right-hand side is a free $K^{0}\left(\mathbb{P}_{0}^{\infty}(\mathbb{C}), \mathbb{Z}_{p}\right)$-module of rank one generated by the standard Thom class $\lambda_{-s L}$ of the virtual bundle $-s L$. Moreover, the product

$$
K^{-2 s}\left(\mathbb{P}_{-s}^{\infty}(\mathbb{C}), \mathbb{Z}_{p}\right) \otimes K^{-2 s^{\prime}}\left(\mathbb{P}_{-s^{\prime}}^{\infty}(\mathbb{C}), \mathbb{Z}_{p}\right) \rightarrow K^{-2\left(s+s^{\prime}\right)}\left(\mathbb{P}_{-\left(s+s^{\prime}\right)}^{\infty}(\mathbb{C}), \mathbb{Z}_{p}\right)
$$

takes $\lambda_{-s L} \otimes \lambda_{-s^{\prime} L}$ to $\lambda_{-\left(s+s^{\prime}\right) L}$. This defines the generator $\lambda_{-L}$. We define the generator $h=[L]-1 \in K^{0}\left(\mathbb{P}_{0}^{\infty}(\mathbb{C}), \mathbb{Z}_{p}\right)$ to be the class of the reduced canonical line bundle. Let $\hat{\beta} \in K^{-2}\left(\mathbb{P}_{-1}^{\infty}(\mathbb{C}), \mathbb{Z}_{p}\right)$ be the image of the Bott element $\beta$ by the composite of the canonical maps

$$
K^{-2}\left(S^{0}, \mathbb{Z}_{p}\right) \stackrel{\gamma}{\rightarrow} K^{-2}\left(\mathbb{P}_{0}^{\infty}(\mathbb{C}), \mathbb{Z}_{p}\right) \rightarrow K^{-2}\left(\mathbb{P}_{-1}^{\infty}(\mathbb{C}), \mathbb{Z}_{p}\right)
$$

The class $\hat{\beta}$ is represented in the spectral sequence by the element $\beta$ and the product

$$
K^{0}\left(\mathbb{P}_{0}^{\infty}(\mathbb{C}), \mathbb{Z}_{p}\right) \otimes K^{-2}\left(\mathbb{P}_{-1}^{\infty}(\mathbb{C}), \mathbb{Z}_{p}\right) \rightarrow K^{-2}\left(\mathbb{P}_{-1}^{\infty}(\mathbb{C}), \mathbb{Z}_{p}\right)
$$

takes $([L]-1) \otimes \lambda_{-L}$ to $\hat{\beta}$.

Proposition 3.1.1. The graded ring

$$
\pi_{*}\left(\hat{\mathbb{H}}\left(\mathbb{T}, f^{*} k u\right)\right)=\bigoplus_{s \in \mathbb{Z}} K^{-2 s}\left(\mathbb{P}_{-s}^{\infty}(\mathbb{C}), \mathbb{Z}_{p}\right)
$$

is the tensor product of the power series algebra and the Laurent polynomial algebra over $\mathbb{Z}_{p}$ generated by the class $[L]-1 \in K^{0}\left(\mathbb{P}_{0}^{\infty}(\mathbb{C}), \mathbb{Z}_{p}\right)$ of the reduced canonical line bundle and by the standard Thom class $\lambda_{-L} \in K^{-2}\left(\mathbb{P}_{-1}^{\infty}(\mathbb{C}), \mathbb{Z}_{p}\right)$ of negative the canonical line bundle, respectively. Moreover

$$
\hat{\beta}=([L]-1) \cdot \lambda_{-L} .
$$

3.2. It follows from theorems of Suslin [1, 12 that the spectrum $k u$ and the $p$-completion of the algebraic $K$-theory spectrum $K(\bar{K})$ are weakly equivalent ring spectra and that we can choose a weak equivalence of ring spectra

$$
\iota_{\varepsilon}: k u \stackrel{\sim}{\longrightarrow} K(\bar{K})^{\wedge}
$$


such that the induced map of homotopy groups takes the Bott element $\beta$ to the Bott element $\beta_{\varepsilon}$. We obtain a map of $\mathbb{T}$-ring spectra

$$
\tau_{\varepsilon}: f^{*} k u \rightarrow T(\bar{V} \mid \bar{K})^{\wedge}
$$

as the adjoint of the composite of the map $\iota_{\varepsilon}$ and the trace map

$$
\operatorname{tr}: K(\bar{K})^{\wedge} \rightarrow f_{*} T(\bar{V} \mid \bar{K})^{\wedge} \text {. }
$$

We also recall from Lemma 2.1.1 that the canonical map

$$
\hat{\mathbb{H}}(\mathbb{T}, T(\bar{V} \mid \bar{K}))^{\wedge} \rightarrow \underset{F}{\operatorname{holim}} \hat{\mathbb{H}}\left(C_{p^{n}}, T(\bar{V} \mid \bar{K})\right)^{\wedge}
$$

is a weak equivalence and from Prop. 2.1.4 that the map

$$
\hat{\Gamma}_{n}: \operatorname{TR}^{n}(\bar{V} \mid \bar{K} ; p)^{\wedge} \rightarrow \hat{\mathbb{H}}\left(C_{p^{n}}, T(\bar{V} \mid \bar{K})\right)^{\wedge}
$$

induces an isomorphism of homotopy groups in non-negative degrees. Moreover, the definition of the map $\hat{\Gamma}_{n}$, 8 Sect. 1.1] shows that $F \circ \hat{\Gamma}_{n}=\hat{\Gamma}_{n-1} \circ F$. Hence there is a well-defined multiplicative map in the stable homotopy category

$$
\hat{\Gamma}: \operatorname{TF}(\bar{V} \mid \bar{K} ; p)^{\wedge}=\operatorname{holim}_{F} \operatorname{TR}^{n}(\bar{V} \mid \bar{K} ; p)^{\wedge} \rightarrow \hat{\mathbb{H}}(\mathbb{T}, T(\bar{V} \mid \bar{K}))^{\wedge}
$$

which induces an isomorphism of homotopy groups in non-negative degrees. Let

$$
\hat{\tau}_{\varepsilon}: \hat{\mathbb{H}}\left(\mathbb{T}, f^{*} k u\right) \rightarrow \hat{\mathbb{H}}\left(\mathbb{T}, T(\bar{V}, \bar{K})^{\wedge}\right)
$$

be the map of $\mathbb{T}$-Tate spectra induced from the map $\tau_{\varepsilon}$.

Lemma 3.2.1. The map $\hat{\tau}_{\varepsilon}$ takes the class $\hat{\beta}$ to the class $\hat{\Gamma}\left(\beta_{\varepsilon}\right)$.

Proof. We recall [8 Sect. 1.1] the following commutative diagram of spectra and multiplicative maps.

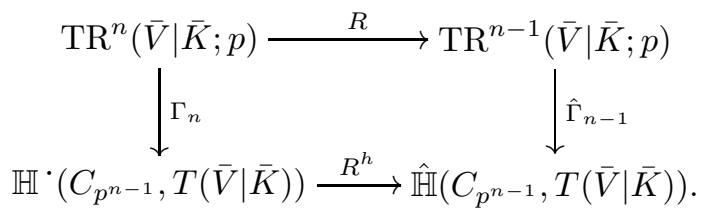

All maps in this diagram are compatible with the respective Frobenius maps. Hence, we obtain a well-defined commutative diagram of spectra and multiplicative maps in the stable homotopy category

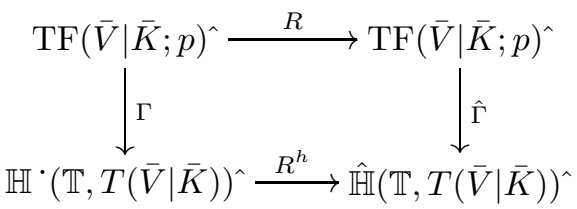

and Prop. 2.1.4 shows that the vertical maps induce isomorphisms of homotopy groups in non-negative degrees. The Bott element $\hat{\beta}$ is defined to be the image of the Bott element $\beta=\beta^{k u}$ by the map of homotopy groups induced by the following composite map

$$
k u \stackrel{\gamma}{\rightarrow} \mathbb{H} \cdot\left(\mathbb{T}, f^{*} k u\right) \stackrel{R^{h}}{\rightarrow} \hat{\mathbb{H}}\left(\mathbb{T}, f^{*} k u\right) .
$$


Moreover, the following diagram commutes

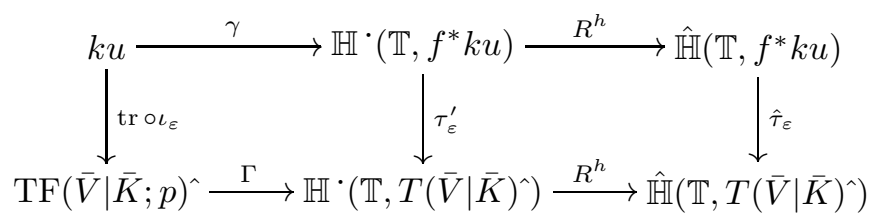

The map of homotopy groups induced by the left-hand vertical map takes the Bott element $\beta=\beta^{k u}$ to the Bott element $\beta_{\varepsilon}=\beta_{\varepsilon}^{\mathrm{TF}}$. Since the restriction map

$$
R: \mathrm{TF}_{2}\left(\bar{V} \mid \bar{K} ; p, \mathbb{Z}_{p}\right) \rightarrow \mathrm{TF}_{2}\left(\bar{V} \mid \bar{K} ; p, \mathbb{Z}_{p}\right)
$$

fixes $\beta_{\varepsilon}$, and since the diagram (3.2.2) commutes, the composition of the lower horizontal maps in the diagram above takes the Bott element $\beta_{\varepsilon}$ to the class $\hat{\Gamma}\left(\beta_{\varepsilon}\right)$. The lemma follows.

Lemma 3.2.3. The map $\hat{\tau}_{\varepsilon}$ takes the class $[L]$ to the class $\hat{\Gamma}\left(\psi\left(\left[\varepsilon_{1}^{\prime}\right]\right)\right)$ for a possibly different sequence $\varepsilon^{\prime}=\left\{\varepsilon^{\prime(n)}\right\}_{n \geq 1}$ of primitive $p^{n-1}$ th roots of unity in $\bar{V}^{\wedge}$.

Proof. We recall from Addendum 1.2.4 that the restriction map

$$
R: \mathrm{TF}_{0}\left(\bar{V} \mid \bar{K} ; p, \mathbb{Z}_{p}\right) \rightarrow \mathrm{TF}_{0}\left(\bar{V} \mid \bar{K} ; p, \mathbb{Z}_{p}\right)
$$

takes $\psi\left(\left[\varepsilon^{\prime}\right]\right)$ to $\psi\left(\left[\varepsilon_{1}^{\prime}\right]\right)$. Hence, in view of the diagram (3.2.2), it suffices to prove that the map of homotopy groups induced by the map

$$
\tau_{\varepsilon}^{\prime}: \mathbb{H} \cdot\left(\mathbb{T}, f^{*} k u\right) \rightarrow \mathbb{H}^{\cdot}\left(\mathbb{T}, T(\bar{V} \mid \bar{K})^{\wedge}\right)
$$

takes the class $[L]$ to $\Gamma\left(\psi\left(\left[\varepsilon^{\prime}\right]\right)\right)$. Let

$$
\tau_{\varepsilon, n}^{\prime}: \mathbb{H}^{\cdot}\left(C_{p^{n-1}}, f^{*} k u\right) \rightarrow \mathbb{H}^{\cdot}\left(C_{p^{n-1}}, T(\bar{V} \mid \bar{K})^{\wedge}\right)
$$

be the map induced by the map $\tau_{\varepsilon}$. The maps $\tau_{\varepsilon, n}^{\prime}$ are compatible with the Frobenius maps such that we have a map of pro-spectra

$$
\left\{\tau_{\varepsilon, n}^{\prime}\right\}_{n \geq 1}:\left\{\mathbb{H} \cdot\left(C_{p^{n-1}}, f^{*} k u\right)\right\}_{n \geq 1} \rightarrow\left\{\mathbb{H}^{\cdot}\left(C_{p^{n-1}}, T(\bar{V} \mid \bar{K})^{\wedge}\right)\right\}_{n \geq 1} .
$$

Moreover, there is a commutative diagram of spectra

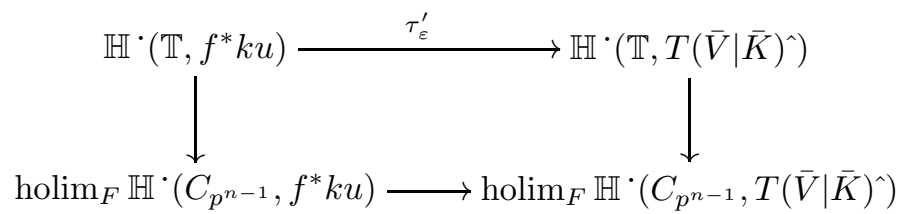

where the lower horizontal map is induced by the map of pro-spectra $\left\{\tau_{\varepsilon, n}^{\prime}\right\}$. The vertical maps are weak equivalences by the proof of Lemma 2.1.1. The Atiyah-Segal completion theorem shows that as a ring

$$
\pi_{0}\left(\mathbb{H} \cdot\left(C_{p^{n-1}}, f^{*} k u\right)\right)=\mathbb{Z}_{p}\left[t_{n}\right] /\left(t_{n}^{p^{n-1}}-1\right)
$$

where $t_{n}$ is the image of $[L]$, and Prop. 2.1.4 shows that the ring homomorphism

$$
\Gamma_{n}: W_{n}(\bar{V})^{\wedge} \rightarrow \pi_{0}\left(\mathbb{H} \cdot\left(C_{p^{n-1}}, T(\bar{V} \mid \bar{K})^{\wedge}\right)\right)
$$

is an isomorphism. Hence the map of pro-spectra $\left\{\tau_{\varepsilon, n}^{\prime}\right\}$ induces a map of pro-rings

$$
\tilde{a}=\left\{\tilde{a}^{(n)}\right\}_{n \geq 1}:\left\{\mathbb{Z}_{p}\left[t_{n}\right] /\left(t_{n}^{p^{n-1}}\right)\right\}_{n \geq 1} \rightarrow\left\{W_{n}(\bar{V})^{\wedge}\right\}_{n \geq 1} .
$$


The map $\tilde{a}$ determines and is determined by the sequence $a=\left\{a^{(n)}\right\}_{n \geq 1}$, where $a^{(n)}=\tilde{a}^{(n)}\left(t_{n}\right)$ is a $p^{n-1}$ th root of unity in $W_{n}(\bar{V})^{\wedge}$ and where $F\left(a^{(n)}\right)=a^{(n-1)}$. We claim that for all $n \geq 1$, the map $\tilde{a}^{(n)}$ is injective or equivalently that $a^{(n)}$ is a primitive $p^{n-1}$ th root of unity. Indeed, the map $\tau_{\varepsilon, n}^{\prime}$ induces a map from the spectral sequence

$$
E_{s, t}^{2}=H^{-s}\left(C_{p^{n-1}}, \pi_{t}(k u)\right) \Rightarrow \pi_{s+t}\left(\mathbb{H}^{\cdot}\left(C_{p^{n-1}}, f^{*} k u\right)\right)
$$

to the spectral sequence

$$
E_{s, t}^{2}=H^{-s}\left(C_{p^{n-1}}, T(\bar{V} \mid \bar{K})^{\wedge}\right) \Rightarrow \pi_{s+t}\left(\mathbb{H}^{\cdot}\left(C_{p^{n-1}}, T(\bar{V} \mid \bar{K})^{\wedge}\right)\right) .
$$

The map of $E^{2}$-terms is injective and, for degree reasons, all differentials in the two spectral sequences are zero, so the induced map of $E^{\infty}$-terms is also injective. Hence the induced map of homotopy groups is injective as claimed. Finally, Lemma 1.3.4 shows that $a=\left\{\left[\varepsilon^{\prime(n)}\right]_{n}\right\}_{n \geq 1}$, where $\varepsilon^{\prime}=\left\{\varepsilon^{\prime(n)}\right\}_{n \geq 1}$ is a sequence of primitive $p^{n-1}$ th roots of unity in $\bar{V}^{\wedge}$ such that $\left(\varepsilon^{\prime(n)}\right)^{p}=\varepsilon^{\prime(n-1)}$. The statement follows since the isomorphism $\psi$ of Addendum 1.2 .4 takes $\left[\varepsilon^{\prime}\right]$ to $\left\{\left[\varepsilon^{\prime(n)}\right]_{n}\right\}_{n \geq 1}$.

REMARK 3.2.4. We expect that the two sequences $\varepsilon^{\prime}$ and $\varepsilon$ in the statement of Lemma 3.2 .3 are equal.

Proof of Prop. 2.4.2, We recall from the proof of Prop. 2.3.2 that the Tate spectral sequence associated with the $\mathbb{T}$-spectrum $T(\bar{V} \mid \bar{K})$ takes the form

$$
\hat{E}^{2}=S_{\bar{V}^{\wedge}}\left\{t^{ \pm 1}, \kappa\right\} \Rightarrow \pi_{*}\left(\hat{\mathbb{H}}(\mathbb{T}, T(\bar{V} \mid \bar{K})), \mathbb{Z}_{p}\right)
$$

where the generators $t$ and $\kappa$ are located in bi-degrees $(-2,0)$ and $(2,0)$. Since the non-zero groups are concentrated in even total degree, all differentials are zero. It follows that any class $\alpha \in \mathrm{TF}_{2}\left(\bar{V} \mid \bar{K} ; p, \mathbb{Z}_{p}\right)$ whose image by the map

$$
\hat{\Gamma}: \operatorname{TF}(\bar{V} \mid \bar{K} ; p)^{\wedge} \rightarrow \hat{\mathbb{H}}(\mathbb{T}, T(\bar{V} \mid \bar{K}))^{\wedge}
$$

represents $t^{-1} \in \hat{E}_{2,0}^{2}$ is a $\mathrm{TF}_{0}\left(\bar{V} \mid \bar{K} ; p, \mathbb{Z}_{p}\right)$-module generator. Let

$$
\alpha \in \lim _{F} T_{p} W_{n} \Omega_{(\bar{V}, \bar{M})}^{1} \stackrel{\sim}{\rightarrow} \mathrm{TF}_{2}\left(\bar{V} \mid \bar{K} ; p, \mathbb{Z}_{p}\right)
$$

to be the unique class such that $\hat{\Gamma}(\alpha)=\hat{\tau}_{\varepsilon}\left(\lambda_{-L}\right)$. Since $\hat{\beta}=([L]-1) \cdot \lambda_{-L}$ by Prop. 3.1.1 and since Lemmas 3.2.1 and 3.2.3 show that the composite

$$
\pi_{2}\left(\hat{\mathbb{H}}\left(\mathbb{T}, f^{*} k u\right)\right) \stackrel{\hat{\tau}_{\varepsilon}}{\longrightarrow} \pi_{2}\left(\hat{\mathbb{H}}(\mathbb{T}, T(\bar{V} \mid \bar{K})), \mathbb{Z}_{p}\right) \stackrel{\sim}{\leftarrow} \mathrm{TF}_{2}\left(\bar{V} \mid \bar{K} ; p, \mathbb{Z}_{p}\right)
$$

takes $([L]-1) \cdot \lambda_{-L}$ to $\psi\left(\left[\varepsilon_{1}^{\prime}\right]-1\right) \cdot \alpha$ and $\hat{\beta}$ to $\beta_{\varepsilon}=\beta_{\varepsilon}^{\mathrm{TF}}$, we find that

$$
\psi\left(\left[\varepsilon_{1}^{\prime}\right]-1\right) \cdot \alpha=\beta_{\varepsilon} .
$$

We claim that $u=\left(\left[\varepsilon_{1}^{\prime}\right]-1\right) /\left(\left[\varepsilon_{1}\right]-1\right)$ is a unit in $W\left(R_{\bar{V}}\right)$. Indeed, this is the case if and only if the image $\bar{u}=\left(\varepsilon_{1}^{\prime}-1\right) /\left(\varepsilon_{1}-1\right)$ by the canonical projection $W\left(R_{\bar{V}}\right) \rightarrow R_{\bar{V}}$ is a unit in $R_{\bar{V}}$. But $R_{\bar{V}}$ is a valuation ring and

$$
v_{R}(\bar{u})=v_{R}\left(\varepsilon_{1}^{\prime}-1\right)-v_{R}(\varepsilon-1)=\frac{1}{p-1}-\frac{1}{p-1}=0,
$$

so $\bar{u}$ is a unit. Hence $\alpha_{\varepsilon}=\psi(u) \cdot \alpha$ is a generator of $\operatorname{TF}_{2}\left(\bar{V} \mid \bar{K} ; p, \mathbb{Z}_{p}\right)$ and the formula $\beta_{\varepsilon}=\psi\left(\left[\varepsilon_{1}\right]-1\right) \cdot \alpha_{\varepsilon}$ holds as desired. 


\section{Galois invariants}

4.1. We show in Prop. 4.1.3 below that in positive degrees, the rational homotopy groups $\operatorname{TR}_{q}^{n}\left(\bar{V} \mid \bar{K} ; p, \mathbb{Q}_{p}\right)$ have vanishing Galois cohomology.

Lemma 4.1.1. For every ring $A$, the ghost map induces an isomorphism

$$
w \otimes 1: W_{n}(A) \otimes \mathbb{Z}\left[\frac{1}{p}\right] \stackrel{\sim}{\rightarrow} A^{n} \otimes \mathbb{Z}\left[\frac{1}{p}\right] .
$$

Proof. Suppose first that $A$ is $p$-torsion free ring, possibly without unit. Then the ghost map restricts to an isomorphism

$$
w W_{n}(p A) \stackrel{\sim}{\rightarrow} \prod_{s=0}^{n-1} p^{s+1} A .
$$

Hence we have a map of short-exact sequences

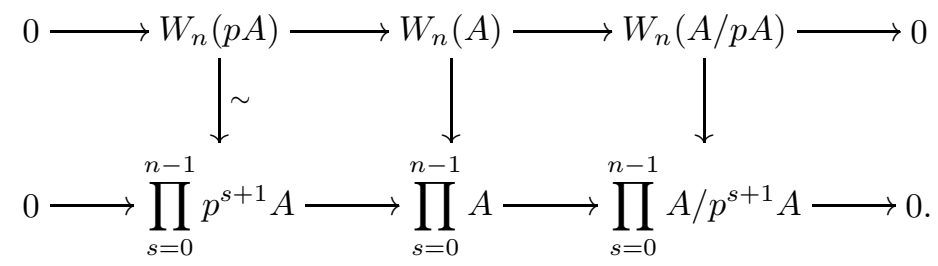

Since $\mathbb{Z}\left[\frac{1}{p}\right]$ is flat over $\mathbb{Z}$, we get an induced map of short-exact sequences

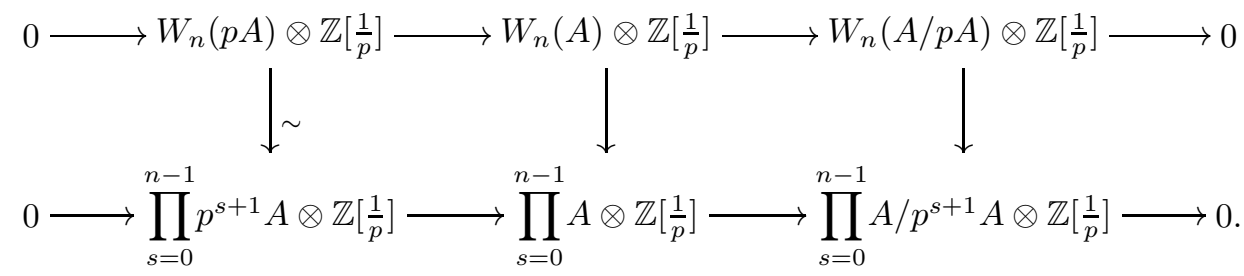

The right hand terms are both zero. Hence, the middle vertical map is an isomorphism. Finally, for a general ring $A$, we write $A=P / I$ where $P$, and hence $I$, is a ring without $p$-torsion. Then the diagram

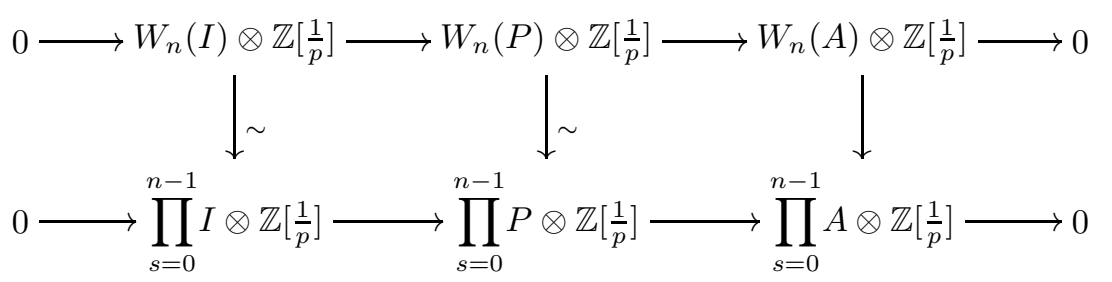

completes the proof.

Lemma 4.1.2. For all $n, q \geq 1$, the canonical inclusion

$$
W_{n}(\bar{V})^{\wedge} \cdot \beta_{\varepsilon, n}^{q} \rightarrow W_{n}(\bar{V})^{\wedge} \cdot \alpha_{\varepsilon, n}^{q}
$$

becomes an isomorphism after inverting $p$.

Proof. By Lemma 4.1.1 the ghost map induces an isomorphism

$$
w W_{n}(\bar{V})^{\wedge}\left[\frac{1}{p}\right] \stackrel{\sim}{\rightarrow} \prod_{i=0}^{n-1} \bar{K}^{\wedge}
$$


And since $\beta_{\varepsilon, n}=\theta_{n}\left(\left[\varepsilon_{n}\right]-1\right) \cdot \alpha_{\varepsilon, n}$, it suffices to show that ghost map takes the element $\theta_{n}\left(\left[\varepsilon_{n}\right]-1\right)=\left[\varepsilon_{1}^{(n)}\right]_{n}-1$ to a unit. But

$$
w\left(\left[\varepsilon_{1}^{(n)}\right]_{n}-1\right)=\left(\left[\varepsilon_{1}^{(n)}\right]_{n}-1,\left[\varepsilon_{1}^{(n)}\right]_{n}^{p}-1, \ldots,\left[\varepsilon_{1}^{(n)}\right]_{n}^{p^{n-1}}-1\right),
$$

and since $\varepsilon_{1}^{(n)}$ is a primitive $p^{n}$ th root of unity, the ghost coordinates are all nonzero.

Proposition 4.1.3. Let $q$ be a positive integer. Then the continuous cohomology group $H_{\text {cont }}^{i}\left(G_{K}, \operatorname{TR}_{q}^{n}\left(\bar{V} \mid \bar{K} ; p, \mathbb{Q}_{p}\right)\right)$ is zero, for all $i \geq 0$.

Proof. By Lemma 4.1.2 we have a canonical isomorphism of $G_{K}$-modules

$$
W_{n}(\bar{V})^{\wedge} \otimes_{\mathbb{Z}_{p}} \mathbb{Q}_{p}(m) \stackrel{\sim}{\rightarrow} \mathrm{TR}_{2 m}^{n}\left(\bar{V} \mid \bar{K} ; p, \mathbb{Q}_{p}\right) .
$$

The $V$-filtration of the left-hand side is finite of length $n$, and

$$
\operatorname{gr}_{V}^{s} W_{n}(\bar{V})^{\wedge} \otimes_{\mathbb{Z}_{p}} \mathbb{Q}_{p}(m)=\bar{K}^{\wedge}(m) .
$$

But Tate 13 has shown that $H_{\text {cont }}^{i}\left(G_{K}, \bar{K}^{\wedge}(m)\right)$ is zero, for all $i \geq 0$, provided that $m>0$.

\section{References}

[1] E. Artin, Algebraic numbers and algebraic functions. I., Institute for Mathematics and Mechanics, New York University, New York, 1951.

[2] J. M. Boardman, Conditionally convergent spectral sequences, Contemp. Math., vol. 239, Amer. Math. Soc., Providence, RI, 1999.

[3] A. K. Bousfield, The localization of spectra with respect to homology, Topology 18 (1979), $257-281$.

[4] J.-M. Fontaine, Le corps des périodes p-adiques, Périodes $p$-adiques (Séminaire de Bures, 1988), Astérisque, vol. 223, 1994, pp. 59-111.

[5] J. P. C. Greenlees, Representing Tate cohomology of G-spaces, Proc. Edinburgh Math. Soc. (2) 30 (1987), 435-443.

[6] L. Hesselholt, Algebraic K-theory and trace invariants, Proceedings of the International Congress of Mathematicians, Vol. II (Beijing, 2002), Higher Ed. Press, Beijing, 2002, pp. 415-425.

[7] L. Hesselholt and I. Madsen, On the K-theory of finite algebras over Witt vectors of perfect fields, Topology 36 (1997), 29-102.

[8] - On the K-theory of nilpotent endomorphisms, Homotopy methods in algebraic topology (Boulder, CO, 1999), Contemp. Math., vol. 271, Amer. Math. Soc., Providence, RI, 2001, pp. $127-140$.

[9] - On the de Rham-Witt complex in mixed characteristic, Ann. Sci. École Norm. Sup. 37 (2004), 1-43.

[10] L. G. Lewis, J. P. May, and M. Steinberger, Equivariant stable homotopy theory, Lecture Notes in Math., vol. 1213, Springer-Verlag, New York, 1986.

[11] A. A. Suslin, On the K-theory of algebraically closed fields, Invent. Math. 73 (1983), 241-245.

[12] _ On the K-theory of local fields, J. Pure Appl. Alg. 34 (1984), 304-318.

[13] J. Tate, p-divisible groups, Proc. Conf. Local Fiels (Driebergen, 1966), Springer-Verlag, Berlin, 1967, pp. 158-183.

Massachusetts Institute of Technology, Cambridge, Massachusetts

E-mail address: larsh@math.mit.edu

NAgOYA University, NAGOYA, JAPAN

E-mail address: larsh@math.nagoya-u.ac.jp 